\title{
Reproductive age-associated fibrosis in the stroma of the mammalian ovary
}

\author{
Shawn M Briley ${ }^{1, *}$, Susmita Jasti ${ }^{1}{ }^{*}$, Jennifer M McCracken², Jessica E Hornick ${ }^{3}$, \\ Barbara Fegley ${ }^{1,4}$, Michele T Pritchard² and Francesca E Duncan ${ }^{1,+}$ \\ ${ }^{1}$ Department of Anatomy and Cell Biology, University of Kansas Medical Center, Kansas City, Kansas, USA, \\ ${ }^{2}$ Department of Pharmacology, Toxicology, and Therapeutics, University of Kansas Medical Center, Kansas City, \\ Kansas, USA, ${ }^{3}$ Biological Imaging Facility, Northwestern University, Evanston, Illinois, USA and ${ }^{4}$ Electron Microscopy \\ Research Laboratory, University of Kansas Medical Center, Kansas City, Kansas, USA
}

Correspondence should be addressed to F E Duncan; Email: f-duncan@northwestern.edu

${ }^{\dagger}$ (F E Duncan is currently at Department of Obstetrics and Gynecology, Northwestern University, Chicago, Illinois, USA) *(S M Briley and S Jasti contributed equally to this work)

\begin{abstract}
Under normal physiological conditions, tissue remodeling in response to injury leads to tissue regeneration without permanent damage. However, if homeostasis between synthesis and degradation of extracellular matrix (ECM) components is altered, fibrosis or the excess accumulation of ECM - can disrupt tissue architecture and function. Several organs, including the heart, lung and kidney, exhibit age-associated fibrosis. Here we investigated whether fibrosis underlies aging in the ovary - an organ that ages chronologically before other organs. We used Picrosirius Red (PSR), a connective tissue stain specific for collagen I and III fibers, to evaluate ovarian fibrosis. Using bright-field, epifluorescence, confocal and polarized light microscopy, we validated the specific staining of highly ordered PSR-stained fibers in the ovary. We next examined ovarian PSR staining in two mouse strains (CD1 and CB6F1) across an aging continuum and found that PSR staining was minimal in ovaries from reproductively young adult animals, increased in distinct foci in animals of mid-to-advanced reproductive age, and was prominent throughout the stroma of the oldest animals. Consistent with fibrosis, there was a reproductive age-associated increase in ovarian hydroxyproline content. We also observed a unique population of multinucleated macrophage giant cells, which are associated with chronic inflammation, within the ovarian stroma exclusively in reproductively old mice. In fact, several genes central to inflammation had significantly higher levels of expression in ovaries from reproductively old mice relative to young mice. These results establish fibrosis as an early hallmark of the aging ovarian stroma, and this altered microenvironment may contribute to the age-associated decline in gamete quality.

Reproduction (2016) 152 245-260
\end{abstract}

\section{Introduction}

The female reproductive system is the first organ system to show overt signs of physiologic aging in the human and is characterized by a marked reduction in both gamete quantity and quality beginning when women reach their mid-thirties - a phenomenon referred to as the maternal age effect (Broekmans et al. 2009). Reproductive aging is associated with aneuploidy, miscarriages, birth defects and infertility, and such consequences are a significant societal concern as more women are globally delaying childbearing (Heffner 2004, Johnson et al. 2012, Nagaoka et al. 2012). Although all women experience reproductive aging, the mechanisms underlying this phenomenon are not fully elucidated.

Data from Assisted Reproduction Technologies (ART) demonstrate that the age-associated decline in fertility is primarily attributable to defects at the level of the gamete. For example, when women of advanced reproductive age conceive using donor oocytes from young individuals, live birth outcomes reflect the age of the donor (Check et al. 2011). In effect, donor oocytes from young women rescue the maternal age effect. Whether the maternal age effect is due to gamete intrinsic or extrinsic factors, or a combination of both, is unknown. Oocytes do not develop in isolation but rather they are surrounded by companion somatic cells within the context of ovarian follicles, which themselves develop within and depend upon a complex stromal microenvironment. The ovarian milieu is composed of a structural network of extracellular matrix (ECM) components and a heterogeneous array of fibroblasts, smooth muscle cells, endothelial cells, theca-interstitial cells and immune cells. This ovarian microenvironment can have a significant impact on follicle and oocyte quality. For example, using a mouse model of reproductive aging, it has been shown previously that secondary follicles isolated from reproductively old mice have altered 
growth and survival, hormone production profiles, and gamete quality outcomes when grown in vitro relative to follicles isolated from young mice (Hirshfeld-Cytron et al. 2011). These results suggest that there are inherent differences between these follicle cohorts that may arise from the distinct ovarian microenvironments from which they were derived. Thus, understanding how the ovarian stroma changes with advanced reproductive age is of critical importance for better understanding the decline in gamete quality. Of note, it was documented that ovaries from reproductively old mice were more rigid compared to those from reproductively young mice, suggesting the presence of age-associated fibrosis (Hirshfeld-Cytron et al. 2011).

Fibrosis is due to an excessive amount of ECM that replaces parenchymal tissue. ECM is composed of collagens, elastin, glycoproteins and proteoglycans, which comprise connective tissue and basement membranes. Collagen is the main structural protein family of connective tissue and provides structural integrity to tissues in addition to regulating their function through signaling. Changes in the collagen matrix of soft tissues mostly affect the fibrillar type I and III collagens, and type I collagen is the most ubiquitously expressed ECM molecule within the mouse ovary (Berkholtz et al. 2006, Alves et al. 2015). Fibrosis occurs when normal tissue remodeling and wound-healing responses are not properly regulated; this can lead to aberrant tissue architecture and thereby compromised organ function (Wynn \& Ramalingam 2012). Inflammation also plays a fundamental role in fibrosis (Libby 2007, Sziksz et al. 2015). There are several organ systems that become fibrotic with age. For example, collagen accumulates with age in the heart, which leads to fibrosis and contributes to diastolic dysfunction (Horn \& Trafford 2016). In the lung, aging is associated with an increase of chronic lung diseases associated with fibrosis such as idiopathic pulmonary fibrosis and chronic obstructive pulmonary disease (Kapetanaki et al. 2013). In the aging kidney, glomerulosclerosis and interstitial fibrosis result in a decreased glomerular filtration rate (Denic et al. 2016, Sangaralingham et al. 2016). Whether fibrotic changes occur in the mammalian ovary, which ages up to decades prior to other organs in humans, has not been systematically investigated.

Here we characterized and applied Picrosirius Red (PSR) staining to assess ovarian fibrosis during physiologic reproductive aging in two mouse strains. PSR is one of the most important selective histological stains used to study fibrillar collagen networks in tissue sections and is frequently used to evaluate fibrosis (Junqueira et al. 1979, Montes \& Junqueira 1991, Coleman 2011, Lattouf et al. 2014). PSR is an anionic dye comprising six sulfonate groups that can associate along cationic collagen fibers (Coleman 2011, Lattouf et al. 2014). This dye specifically binds to collagen I and III fibrils and is thought to detect more advanced stages of fibrosis (Coleman 2011, Lattouf et al. 2014). We evaluated
PSR staining in fixed paraffin-embedded ovarian tissue sections using four imaging approaches, including brightfield, epifluorescence, confocal and polarized light microscopy, which together demonstrate the specificity and versatility of this stain. We then demonstrated that during reproductive aging, PSR staining begins as discrete foci that expand throughout the ovarian stroma coincident with increased hydroxyproline levels, the presence of multinucleated macrophage giant cells and increased expression of genes involved in inflammation. Taken together, this study provides definitive evidence at the histological, biochemical and gene expression levels of prominent age-associated ovarian fibrosis.

\section{Materials and methods}

\section{Animals and ovarian and liver tissue samples}

Ovarian tissue was harvested from two mouse strains (CD1 and $\mathrm{CB} 6 \mathrm{~F} 1$ ), which exhibit reproductive aging phenotypes including increased egg aneuploidy (Chiang et al. 2010, Merriman et al. 2012, Hornick et al. 2015, Treff et al. 2016). Reproductively young CB6F1 mice (6-12 week old) were obtained from Envigo (Indianapolis, IN, USA), and reproductively old CB6F1 mice (14-17 month old) were obtained from the National Institutes on Aging Aged Rodent Colony (National Institutes of Health, Bethesda, MD, USA). For this strain, these two age cohorts are herein referred to as reproductively "young" and "old," respectively. For each experiment, ovarian tissue from a minimum of 3 animals per age group was examined and representative images are shown. Liver tissue from mice exposed to chronic carbon tetrachloride $\left(\mathrm{CCl}_{4}\right)$ to induce fibrosis was used for validation purposes, and these mice were generated as described previously (Weber et al. 2003, Constandinou et al. 2005, Pritchard \& Nagy 2010). All mice were housed in a controlled barrier facility at the University of Kansas Medical Center's (KUMC) Research Support Facility under constant temperature, humidity and light (12 h light: $12 \mathrm{~h}$ darkness). Food and water were provided ad libitum.

CD1 mice (Envigo, Indianapolis, IN, USA) were bred at the Northwestern University and killed at various ages ranging from 6 weeks to 22 months. Histological ovarian sections from this aging continuum were a generous gift of Dr Teresa K Woodruff (Northwestern University, Chicago, IL, USA). For this strain, the specific animal age used for each experiment is denoted throughout the text. All animal experiments described here were approved by the Institutional Animal Care and Use Committee (KUMC or Northwestern University) and were in accordance with the National Institutes of Health Guidelines.

\section{Tissue processing}

Tissue samples were fixed in either 10\% Formalin (liver) or Modified Davidson's (ovary) (Electron Microscopy Sciences, Hatfield, PA, USA). Liver samples were fixed for 16-18 h and ovarian tissue for up to $24 \mathrm{~h}$ at $4^{\circ} \mathrm{C}$. Samples were 
transferred to $70 \%$ ethanol until processed, dehydrated using an automated tissue processor (Leica Biosystems), embedded in paraffin, and sections were cut at $5 \mu \mathrm{m}$ thickness for all histological applications.

\section{PSR staining}

For PSR staining, tissue sections were deparaffinized in Citrisolv (Thermo Fisher Scientific) and then rehydrated in a series of graded ethanol baths (100, 70 and 30\%). Slides were then immersed in a PSR staining solution prepared by dissolving Sirius Red F3BA (Direct Red 80, C.I. 357.82, SigmaAldrich) in a saturated aqueous solution of picric acid (SigmaAldrich) at $0.1 \% \mathrm{w} / \mathrm{v}$. Slides were incubated in the PSR staining solution for $40 \mathrm{~min}$ at room temperature. The slides were then either incubated in $0.5 \%$ glacial acetic acid (Avantor Performance Materials, Central valley, PA, USA) for a total of four 7-min washes or incubated in 0.05 M hydrochloric acid (Thermo Fisher Scientific) for 90 s. Excess acidified water was carefully wicked away from the tissue sections, and the tissue was rapidly dehydrated in $100 \%$ ethanol (a total of three, 30-s incubations). The slides were cleared in Citrisolv for five minutes and mounted with Cytoseal XYL (Thermo Fisher Scientific). For each independent experiment, all slides used for PSR staining were processed at the same time to minimize variation in staining intensity.

\section{PSR imaging}

Bright-field images were taken with an EVOS FL Auto Cell Imaging system (Thermo Fisher) using a 20 or $40 \times$ objective. To view the entire ovary sections, scans comprising a series of individual images were taken across the tissue and then automatically stitched together using the EVOS software. To quantify the area of ovarian tissue that was positive for PSR staining we followed the protocol as described: http:// rsbweb.nih.gov/ij/docs/examples/stained-sections/index.html (access date February 2016). In brief, for each animal, two non-overlapping images were taken of each section at $20 \times$ magnification using the EVOS FL Auto. Imagel was used to quantify the area of positive PSR staining above a threshold that was set based on the staining in the oldest animal for each mouse strain. This threshold was kept constant for all images analyzed for each particular mouse strain. Sections from a total of $n=3$ young and $n=3$ old CB6F1 mice and a total of $n=22$ CD1 mice across an aging continuum were analyzed.

For epifluorescence imaging, we used the EVOS FL Auto Cell Imaging system equipped with the following LED light cubes: Texas Red (Ex 585/29 nm; Em 624/40 nm), Green Fluorescent Protein (GFP) (Ex 470/22 nm; Em 510/42 nm) and DAPI (Ex $357 / 44$ nm; Em 447/60 nm). To detect collagen, the Texas Red light cube was used and the light settings and exposure times were set for the samples with the most intense PSR staining (21 and 22 months old CD1 ovaries and 14-17 months old CB6F1 ovaries). These settings were then kept constant when imaging all the other ovary sections. The appropriate GFP and DAPI light cube imaging settings were established using an ovarian tissue section that had been processed for immunocytochemistry with known green and blue fluorescence (Supplementary Fig. 1B, see section on supplementary data given at the end of this article). In brief, immunofluorescence with a primary antibody against the oocyte-specific protein, MSY2 (generous gift from R Schultz, University of Pennsylvania, Philadelphia, PA, USA), was performed and detected using a Fluorescein TSA detection kit (PerkinElmer). Nuclei were visualized with Vectashield containing DAPI (4',6-diamidino-2-phenylindole) (Vector Laboratories, Burlingame, CA, USA). The settings optimized with this sample were then kept constant when imaging the PSR-stained ovarian sections. All post-processing adjustments of brightness were done equivalently across images using ImageJ software (Schneider et al. 2012).

PSR-stained tissue sections were also imaged using a Leica SPE confocal configured with a Leica DM550 Q upright microscope with an ACS APO $63 \times / 1.30$ Oil CS objective (Leica Microsystems Inc, Buffalo Grove, IL, USA). The 532 nm solid-state laser was used for excitation and $0.4 \mu \mathrm{m}$ optical sections were taken through each sample.

To analyze the birefringence of ovarian tissue stained with Picrosirius Red, we used circularly polarized light microscopy on an Abrio system on an inverted Nikon Eclipse TE-300 equipped with 20 and $40 \times$ objectives (Pol-scope; Cambridge Research \& Instrumentation, Oosight Imaging System, Woburn, MA, USA). The Abrio software was used to determine the orientation of the samples' birefringence properties.

\section{Hydroxyproline assay}

To quantify the collagen content in ovarian tissue, a hydroxyproline assay was performed as described previously (Reddy \& Enwemeka 1996). In brief, ovaries were hydrolyzed in $100 \mu \mathrm{L}$ of water and $100 \mu \mathrm{L}$ of $12.1 \mathrm{~N}$ hydrochloric acid and incubated at $120^{\circ} \mathrm{C}$ in a dry bath incubator for $3 \mathrm{~h}$. Samples were vortexed every $30 \mathrm{~min}$ during this incubation period. Samples were then centrifuged for $10 \mathrm{~min}$ at $10,000 \mathrm{~g}$. Five and $10 \mu \mathrm{L}$ of the supernatant from the hydrolyzed tissues were transferred to a 96 well plate to which $100 \mu \mathrm{L}$ of Chloramine T reagent (Reddy \& Enwemeka 1996) was added and allowed to incubate at room temperature for $25 \mathrm{~min}$. Then, $100 \mu \mathrm{L}$ of Ehrlich's Solution [1M 4-(dimethylamino)benzaldehyde in 1-propanol/60\% perchloric acid (3:1, v/v)] was added to each well and incubated at $60^{\circ} \mathrm{C}$ for $35 \mathrm{~min}$ in a non-humidified, laboratory oven. After this incubation, the absorbance of all samples at $550 \mathrm{~nm}$ was measured in a microplate reader. A standard curve of known hydroxyproline concentrations was created from which the concentration of hydroxyproline was calculated for each sample. Collagen contains roughly $12.5 \%$ hydroxylated prolines; therefore to extrapolate the amount of collagen that is present in ovarian tissue per mg of tissue we divided the value for the hydroxyproline concentration by $12.5 \%$. For each assay, a total of 4 ovaries was pooled and hydrolyzed for each age group (reproductively young and old CB6F1 females). The assay was repeated four times, with technical replicates included for each. The fold change was calculated by normalizing to the average collagen values from the reproductively young ovaries for each trial. Fibrotic liver from chronically $\mathrm{CCl}_{4}$-exposed mice served as positive controls while liver from vehicle-treated mice served as negative controls for the assays. 


\section{Additional histological staining}

For hematoxylin and eosin ( $\mathrm{H} \& \mathrm{E})$ staining, tissue sections were stained following a standard H\&E staining protocol, cleared with Citrisolv (3-5-min incubations), and mounted with Cytoseal XYL. For Periodic Acid Schiff (PAS) staining, slides were deparaffinized in Citrisolv, rehydrated in graded ethanol baths (100, 95, 80 and 70\%), and washed in RO water. Slides were then immersed in $0.5 \%$ periodic acid (Thermo Fisher Scientific) for $5 \mathrm{~min}$, washed (4-5-min incubations) in RO water, immersed in Schiff's Reagent (Thermo Fisher Scientific) for $15 \mathrm{~min}$, rinsed in running $\mathrm{RO}$ water for $10 \mathrm{~min}$, and counterstained with hematoxylin. Slides were then dehydrated in graded ethanol baths, cleared in Citrisolv and mounted with Cytoseal XYL.

For autofluorescence detection, tissue sections were deparaffinized, rehydrated in graded ethanol baths, and washed in RO water. Slides were then incubated in Trisbuffered saline supplemented with $0.1 \%$ Tween-20 (TBS-T), 3-10-min incubations, and mounted with Vectashield with or without DAPI. All imaging was performed using the EVOS FL Auto Cell Imaging system.

\section{Immunofluorescence and immunohistochemistry}

For detecting macrophages in ovarian tissue sections, immunofluorescence was performed. In brief, tissues sections were deparaffinized in Citrisolv and rehydrated in a series of graded ethanol baths $(100,95,85,70$ and $50 \%$ ). Antigen retrieval was performed using $10 \times$ Reveal Decloaker (Biocare Medical, Concord, CA, USA) according to the manufacturer's instructions. Slides were rinsed in TBS-T, incubated in 30\% hydrogen peroxide (Thermo Fisher Scientific) followed by incubations in avidin and biotin blocking solutions for $15 \mathrm{~min}$ at room temperature (Vector Laboratories, Burlingame, CA, USA). The tissue sections were then incubated in blocking solution $(10 \%$ normal goat serum (Vector Laboratories, Burlingame, CA, USA) and $0.4 \%$ Triton X-100 (Thermo Fisher Scientific) diluted in TBS) for $1 \mathrm{~h}$ at room temperature and then incubated overnight at $4{ }^{\circ} \mathrm{C}$ in primary antibody at 1:50 dilution (rat anti-mouse F4/80, Bio-Rad). Slides were rinsed in TBS-T and incubated in 1:100 dilution of secondary antibody (biotinylated antirat IgG, Vector Laboratories, Burlingame, CA, USA) for $2 \mathrm{~h}$ at room temperature. The slides were rinsed and incubated in $\mathrm{ABC}$ reagent (Vector Laboratories, Burlingame, CA, USA) for $30 \mathrm{~min}$ at room temperature. Signal amplification was performed using the Fluorescein TSA kit according to the manufacturer's instructions (PerkinElmer). The slides were mounted with Vectashield containing DAPI. We used the EVOS FL Auto Imaging automated cell counting software with watershed algorithm to determine the number of F4/80positive cells (fluorescein-positive) cells and total number of cells (DAPI-positive) in ovarian sections. Intensity thresholds were set according to secondary only controls. To determine the percentage of F4/80 positive cells, we reported the average number of fluorescein-positive cells over the total number of cells for each image. A minimum of five images were analyzed for each ovary $(n=3$ reproductively young and $n=3$ reproductively old CB6F1 females).
For detection of alpha-smooth muscle actin ( $\alpha$-SMA), ovarian tissue sections were deparaffinized and antigen retrieval was performed as described previously. Primary and secondary antibody incubations were performed using Vector Mouse on Mouse (M.O.M) kit (Vector Laboratories, Burlingame, CA, USA) and Vector ABC kit (Vector Laboratories, Burlingame, CA, USA) according to the manufacturer's protocols. Detection was done via 3'3'-Diaminobenzidine (DAB) staining using the DAB Peroxidase (HRP) Substrate Kit (Vector Laboratories, Burlingame, CA, USA). The primary antibody used was anti-alpha smooth muscle actin (ab7817) at 1:250 dilution (Abcam). All imaging was performed using the EVOS FL Auto Cell Imaging system.

\section{Electron microscopy}

Ovaries were bisected longitudinally using a scalpel and fixed in $2 \%$ glutaraldehyde in $0.1 \mathrm{M}$ sodium cacodylate buffer. The tissue was transferred to $5 \mathrm{~mL}$ screw cap glass vials for processing. The tissue was rinsed in fresh cacodylate buffer for $20 \mathrm{~min}$ and then post fixed in 1\% buffered osmium tetroxide for $1 \mathrm{~h}$. The samples were rinsed in 3 exchanges of deionized distilled water and then dehydrated through a graded series of ethanols (50, 70, 80, 95 and 100\%). Each step was done for $20 \mathrm{~min}$ and repeated twice. The tissue was placed in propylene oxide for $20 \mathrm{~min}$ and then into a mixture of half volume propylene oxide and half volume Embed 812 medium mixture (Electron Microscopy Sciences, Hatfield, PA, USA), overnight. The half and half mixture was removed the next day and $100 \%$ Embed mixture was added to the sample and allowed to stand for $1 \mathrm{~h}$. Resin was removed and replaced with fresh Embed mixture and placed in a $65^{\circ} \mathrm{C}$ oven to polymerize overnight. Samples were sectioned using a Diatome diamond knife on a Leica UC-7 ultramicrotome. Sections were cut at $1 \mu \mathrm{m}$ until areas of interest were noted and then sections were cut at $70 \mathrm{~nm}$ and picked up on 300 mesh thin bar grids (Electron Microscopy Sciences, Inc) Section contrast was achieved through exposure to $3 \%$ uranyl acetate and Sato's lead stain and viewed with a JEM 1400 TEM (JEOL USA, Inc, Peabody, MA, USA) at $100 \mathrm{kv}$.

\section{Gene expression analysis}

One ovary from each mouse was placed in RNAlater solution (Ambion) to stabilize RNA ( $n=4$ reproductively young and $n=4$ reproductively old CB6F1 females). A bead homogenizer (FastPrep 24, MP Biomedical, Solon, OH, USA) was used to homogenize tissue in RLT buffer (RNeasy Mini Kit, Qiagen) containing $\beta$-mercaptoethanol. The RNeasy Mini kit was used to isolate RNA, and $0.85 \mu \mathrm{g}$ of RNA was reverse transcribed to cDNA using a Retroscript kit (Life Technologies/Ambion). A Bio-Rad CFX384 machine was used for real-time PCR. 185 was used as the housekeeping gene, and gene expression was calculated using the $2^{-\Delta \Delta \mathrm{Ct}}$ method. Results are expressed as fold change in expression over young mice. Primers came from Primer Bank (Wang \& Seed 2003, Spandidos et al. 2008, 2010) unless otherwise stated and include $/ / 1 b$ F: GAAATGCCACCTTTTGACAGTG R: CTGGATGCTCTCATCAGGACA (Primer Bank ID: 118130747b1), //6 F: CTGCAAGAGACTTCCATCCAG 
R: AGTGGTATAGACAGGTCTGTTGG (Primer Bank ID: 13624310b1), $/ 110$ F: GCT CTT ACT GAC TGG CAT GAG R: CGC AGC TCT AGG AGC ATG TG (Primer Bank ID: 6754318A1), CCl2 F: AGGTCCCTGTCATGCTTCTG R: TCTGGACCCATTCCTTCTTG (Pascual et al. 2011), Tnfa F: CCCTCACACTCAGATCATCTTCT R: GCTACGACGTGGGCTACAG (Pritchard et al. 2010), and CCl5 F: TTTGCCTACCTCTCCCTCG R: CGACTGCAAGATTGGAGCACT (Primer Bank ID: 164698427b1). All primer oligonucleotides were synthesized by Integrated DNA Technologies (Coralville, IA, USA) and used at $0.1 \mu \mathrm{M}$ in real-time PCR reactions.

\section{Cytokine antibody arrays}

Ovaries from reproductively young and old CB6F1 mice were harvested, pooled according to age cohort ( $n=4$ mice per age group) and cultured on $0.4 \mu \mathrm{m}, 30 \mathrm{~mm}$ Millicell inserts (Merck Millipore) in 6 well dishes for a total of $2.5 \mathrm{~h}$ in a humidified atmosphere of $5 \% \mathrm{CO}_{2}$ in air at $37^{\circ} \mathrm{C}$. Each well contained $1.5 \mathrm{~mL}$ of culture media, and ovaries were overlaid with a thin film of media. The culture media was $\alpha$ MEM GlutaMAX (Thermo Fisher) supplemented with $0.1 \%$ bovine serum albumin (Thermo Fisher), $1 \times$ ITS-X (ThermoFisher), $0.05 \mathrm{mg} / \mathrm{mL}$ L-ascorbic acid (Sigma-Aldrich) and $1 \times$ PenStrep (Thermo Fisher). Media alone was included as a control. Following culture, $0.5 \mathrm{~mL}$ of the conditioned culture media from each group was combined with $0.5 \mathrm{~mL}$ blocking buffer supplied with the cytokine antibody array kit and then run on the RayBio C-Series Mouse Cytokine Antibody Array C1 according to the manufacturer's protocol (RayBiotech, Norcross, GA, USA). Data analysis was done according to the manufacturer's instructions. In brief, raw numerical densitometry data were extracted from the arrays by measuring the mean integrated density of a defined area for each spot using ImageJ. Background subtraction was performed using the blank values, and positive control normalization was done using the media-only array as a reference. Signals from the mediaonly array were subtracted, and the fold change between young and old samples was calculated. This experiment was repeated twice.

\section{Statistical analysis}

Statistical analyses were performed using Microsoft Excel or GraphPad Prism Software Version 6.0f (La Jolla, CA, USA). For gene expression analysis, a two-tailed $t$-test for significance and a $Q$-test was used to remove any outliers. For other assays, changes between groups were analyzed by Pearson's Correlation or a $t$-test, and a $P$ value $<0.05$ was considered significant.

\section{Results}

\section{Picrosirius Red (PSR) staining reveals collagen networks in the ovary by bright-field microscopy}

Upon binding to collagen fibers, PSR results in a dark red stain that is readily visualized by bright-field microscopy, whereas cytoplasmic regions appear yellow or faint pink (Junqueira et al. 1978, 1979). We first validated our staining conditions using liver samples from mice treated with a chronic $\mathrm{CCl}_{4}$ regimen that is known to induce fibrosis in distinct regions of the liver (Weber et al. 2003, Constandinou et al. 2005, Pritchard \& Nagy 2010). In this model, fibrotic septae connect the central veins throughout the liver in a typical 'chickenwire' pattern characteristic of bridging fibrosis. In control mice, the majority of the liver tissue appeared yellow or light pink, and there was minimal, but expected, PSR staining around the hepatic vessels (Fig. 1A, arrows and asterisks). However, in the $\mathrm{CCl}_{4}$-treated liver samples, there was significant PSR-positive staining surrounding and extending from the central veins (Fig. 1B, asterisks). These results confirmed that we could use PSR staining to distinguish between fibrotic and non-fibrotic tissue.

We next examined PSR staining in ovarian tissue (Fig. 1C). For these characterization studies, we used ovarian tissue from reproductively old mice (21-22 months old), as fibrosis increases with age in several tissues (Kapetanaki et al. 2013, Horn \& Trafford 2016, Sangaralingham et al. 2016). We found a prominent network of intense PSR-stained collagen fibers throughout the ovarian stroma (Fig. 1C). In general, the bulk of the non-fibrotic regions in the ovary stained a darker pink compared to the liver, irrespective of fixation conditions (Fig. 1 and data not shown). Background pink staining may be due to structures that contain basic proteins, suggesting differing tissue compositions between the liver and ovary (Nielsen et al. 1998).

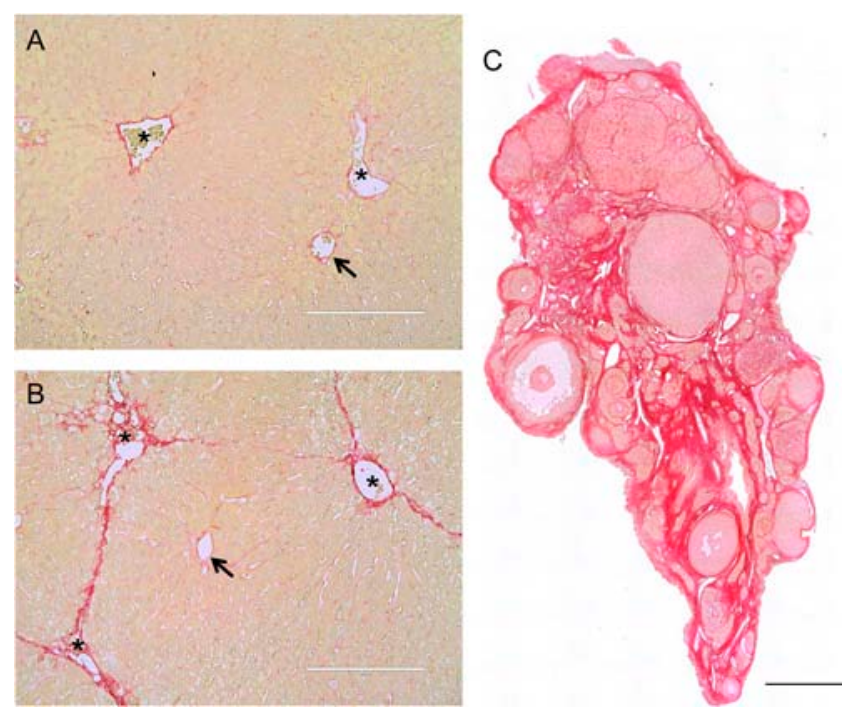

Figure 1 Picrosirius Red staining detects fibrosis. Liver tissue sections from (A) a control mouse injected with vehicle and (B) a mouse injected chronically with $\mathrm{CCl}_{4}$ (2 times per week for 5 weeks). Arrows highlight the hepatic vessels in the periportal areas and asterisks highlight the central vein. (C) An ovarian tissue section from a 21-month old CD1 mouse was stained with PSR using the same protocol. The intense red staining corresponds to fibrotic regions. The scale bars in (A) and (B) are $200 \mu \mathrm{m}$ and $0.4 \mathrm{~mm}$ in (C). 
This background is largely eliminated when visualizing PSR by other methods as described below.

\section{PSR staining exhibits red fluorescence and birefringence that enhances visualization of collagen fibers in the ovary}

In cardiac tissue, PSR-stained collagen fibers produce strong fluorescence in the red wavelength (Dolber \& Spach 1993, Vogel et al. 2015). To examine the fluorescent properties of PSR staining in ovarian tissue, we visualized sections by epifluorescence microscopy. We found that the collagen fibers that stained intense red with PSR by bright-field microscopy corresponded to regions of intense fluorescence when examined with a Texas Red light cube (Fig. 2A, B and Supplementary Fig. 1). This fluorescence was specific to the PSR-stained fibers as no signal was observed when the adjacent unstained sections were examined (Fig. 2B, C, D and Supplementary Fig. 1). Furthermore, PSR-stained tissue only exhibited fluorescence in the near-red wavelength as no appreciable signal was detected when the samples were visualized with either GFP (green wavelength) or DAPI (blue wavelength) light cubes (Supplementary Fig. 1). The red fluorescence signal eliminated the background pink cytoplasmic staining that was prominent in the ovary by bright-field microscopy, further confirming the specificity of PSR for collagen fibers (Figs 1C, 2A, B and Supplementary Fig. 1).

To obtain a more detailed view of the arrangement of the PSR-stained structures within the ovary, we examined histological sections by confocal microscopy. We found that PSR staining detected fibers that ranged from $\sim 5-15 \mu \mathrm{m}$ in diameter throughout the ovary that were composed of linearly arranged bundles of individual fibrils (Fig. 2E and F). This fluorescence was only observed with the $532 \mathrm{~nm}$ laser (Fig. 2E, F and data not shown). These results are consistent with collagen fibers, which are reported to be $1-20 \mu \mathrm{m}$ wide, and therefore provide further validation of the specificity of the PSR staining within the ovary (Ushiki 2002).

To further confirm that PSR detects collagen fibers in the ovary, we imaged PSR-stained ovarian sections using circularly polarized light microscopy. This technique can be used to determine a specimen's optical anisotropy, which is a consequence of its molecular order and architecture (Mehta et al. 2013). Biological structures, such as collagen, are composed of filaments that are anisotropic themselves and can exhibit birefringence or anisotropy of refraction (Mehta et al. 2013). PSR staining enhances the natural birefringence of collagen when examined under polarized light because the PSR dye molecule (F3BA) binds to the tertiary groove of collagen I and III fibrils alongside the fiber axis (Lattouf et al. 2014, Street et al. 2014). Additionally, birefringence is amplified because approximately 120 dye molecules bind per collagen molecule. The signal obtained from
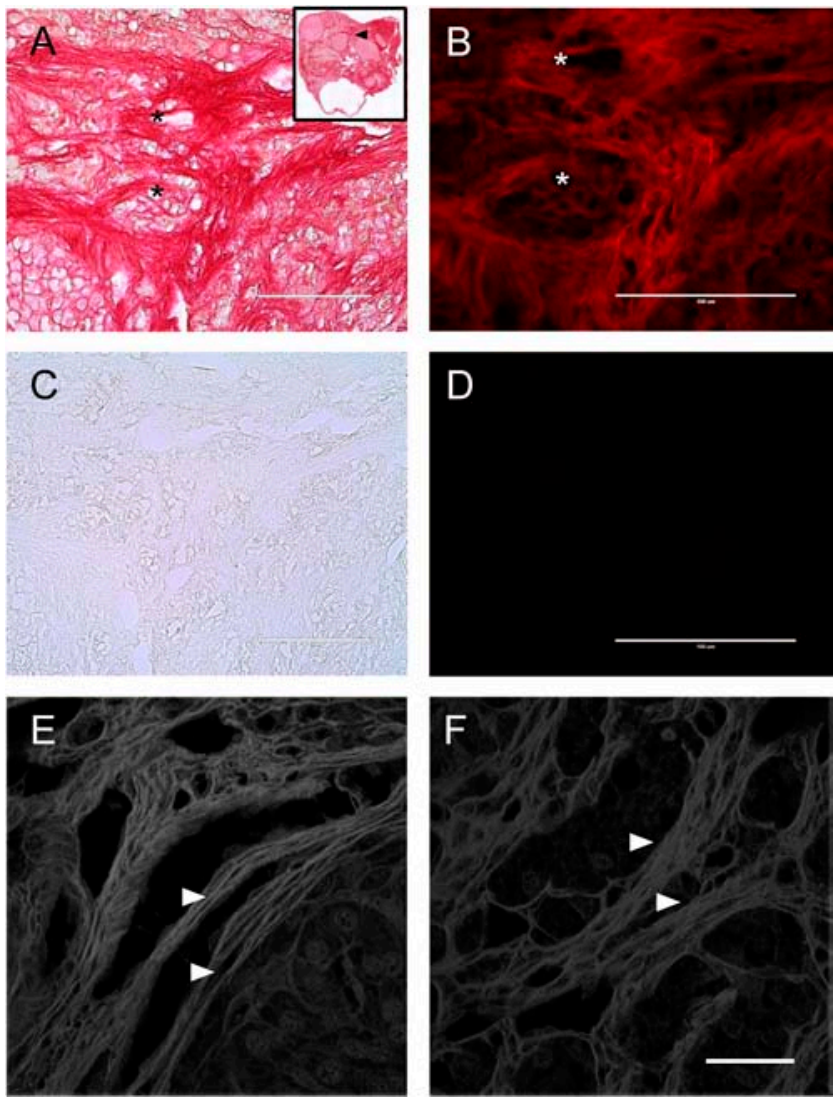

Figure 2 Picrosirius Red staining exhibits red fluorescence and shows fibrils organized into bundles within the ovarian stroma. PSR-stained ovarian tissue from a 22-month old CD1 mouse visualized by (A) bright-field microscopy and (B) epifluorescence microscopy using a Texas Red LED Light Cube. The inset in (A) shows the entire ovarian section, with the region examined in (A, B, C and D) marked by a black arrowhead. An adjacent unstained section was imaged with the same settings by (C) bright-field and (D) epifluorescence microscopy. The asterisks mark corresponding regions between bright-field and fluorescence images. The scale bars are $100 \mu \mathrm{m}$. (E and F) PSRstained ovarian tissue sections from 21- and 22-month old CD1 mice imaged by confocal microscopy using a $532 \mathrm{~nm}$ laser. The white arrowheads highlight fibrils that are organized into larger bundles. Maximum projections of $0.4 \mu \mathrm{m}$-thick optical sections are shown. The scale bar is $25 \mu \mathrm{m}$.

PSR staining, especially when combined with enhanced birefringence, is therefore considered highly specific for collagen (Junqueira et al. 1979, Whittaker et al. 1994, Lattouf et al. 2014, Street et al. 2014). When imaged in this manner, collagen bundles appear bright relative to a black background. Using an Abrio Liquid Crystal (LC) PolScope, we detected the birefringent properties of PSR-stained ovarian tissue sections and found that the highly birefringent regions corresponded to the most intense red staining visualized by bright-field microscopy (Fig. 3A and B), which is consistent with our observations with red fluorescence (Fig. 2B). Moreover, we used the LC-PolScope software to determine the orientation of the molecular order or birefringence in the PSR-stained ovarian tissue. As is evident by the 

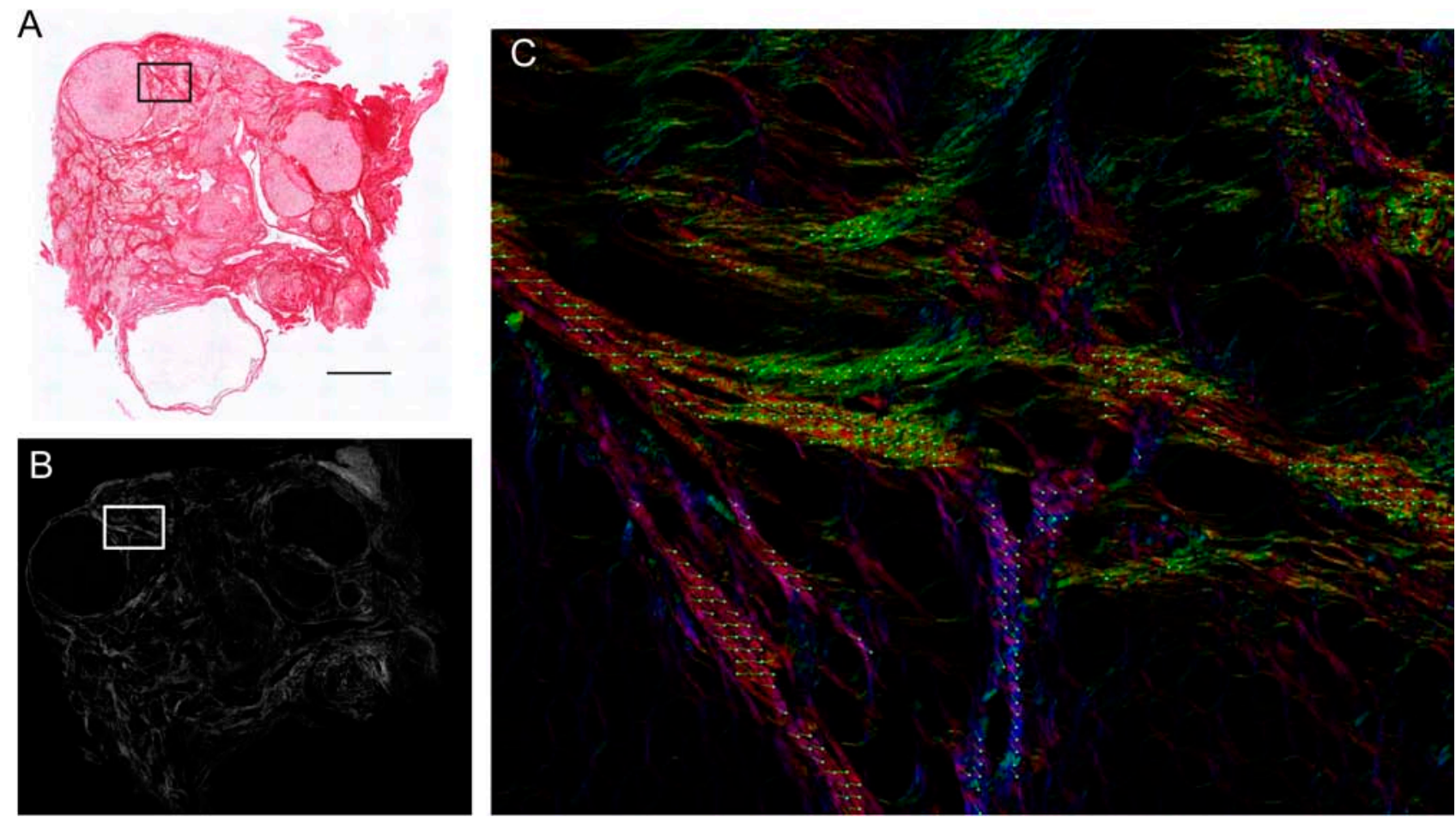

Figure 3 Picrosirius Red fibers in the aged ovary are birefringent. A PSR-stained ovarian tissue section from a 22-month old CD1 mouse visualized by both (A) bright-field microscopy and (B and C) circularly polarized light microscopy (Abrio LC-PolScope). The boxed region in $(\mathrm{A}$ and $\mathrm{B})$ is magnified in $(\mathrm{C})$, where the false color image with retardance vectors (green lines) reflects the orientation of the co-aligned fibers. The scale bar is $0.4 \mathrm{~mm}$.

pseudocolor polarized light image superimposed with retardance vectors, the birefringent PSR-stained collagen bundles are regularly ordered (green retardance vectors facing the same direction) and are arranged with multiple orientations (different colors) in the ovary (Fig. 3C). Taken together, these multiple imaging approaches (bright-field, epifluorescence, confocal and polarized light) provide strong evidence that PSR staining detects highly ordered collagen fibers in the mouse ovary.

\section{PSR staining demonstrates an age-associated increase in fibrosis in the mouse ovary}

Following the validation of PSR staining as an indicator of fibrous collagen in the ovary, we then used this histological tool to evaluate whether fibrosis increased in the mouse ovary with advanced reproductive age. The mouse is an important model for reproductive aging, since several mouse strains exhibit reproductive aging phenotypes that parallel what occurs in human, including loss of the ovarian reserve, altered endocrine function, increased egg aneuploidy, and subfertility (Chiang et al. 2010, Hirshfeld-Cytron et al. 2011, Merriman et al. 2012, Hornick et al. 2015, Treff et al. 2016). We performed PSR staining on an aging series of ovarian tissue sections from mice of the CD1 strain that ranged in age from 6 weeks to 22 months (Fig. 4). In ovaries from animals of all ages, some PSR staining was observed as expected because structures such as follicles, the ovarian surface epithelium, and blood vessels contain collagen (Berkholtz et al. 2006) (Fig. 4). However, fibrotic foci characterized by intense PSR staining visualized using both brightfield and fluorescence microscopy, were visible in the ovarian stroma beginning between 7 and 9 months of age (Fig. 4C, D, 4C', $\mathrm{D}^{\prime}$ and $4 \mathrm{C}^{\prime \prime}, \mathrm{D}^{\prime \prime}$ ). These foci appeared to expand such that fibrosis was prominent throughout the ovarian stroma of animals between 18 and 22 months of age (Fig. 4E, F, 4E', $\mathrm{F}^{\prime}$ and $4 \mathrm{E}^{\prime \prime}$, $\left.F^{\prime \prime}\right)$. In fact, there was a significant positive linear correlation between animal age and the amount of PSR-positive staining observed per ovarian section (Fig. 5A, B, C and D; $P=0.0006$ ).

The reproductive age-associated expansion of PSR staining and fibrosis was not unique to this specific mouse strain as we observed a similar phenomenon within the ovarian stroma of CB6F1 mice as well (Figs 5E, F, G and 6A, B, C, D). Specifically, ovaries from the reproductively old cohort of $\mathrm{CB} 6 \mathrm{~F} 1$ mice had nearly double the amount of PSR-positive staining in the ovarian stroma relative to the reproductively young cohort (Fig. 5E, F and G; 6-12 weeks old vs 14-17 months old; $P=0.03)$. To further quantify this age-associated fibrosis, we compared total collagen levels in ovaries from reproductively young and old CB6F1 mice using a hydroxyproline assay 

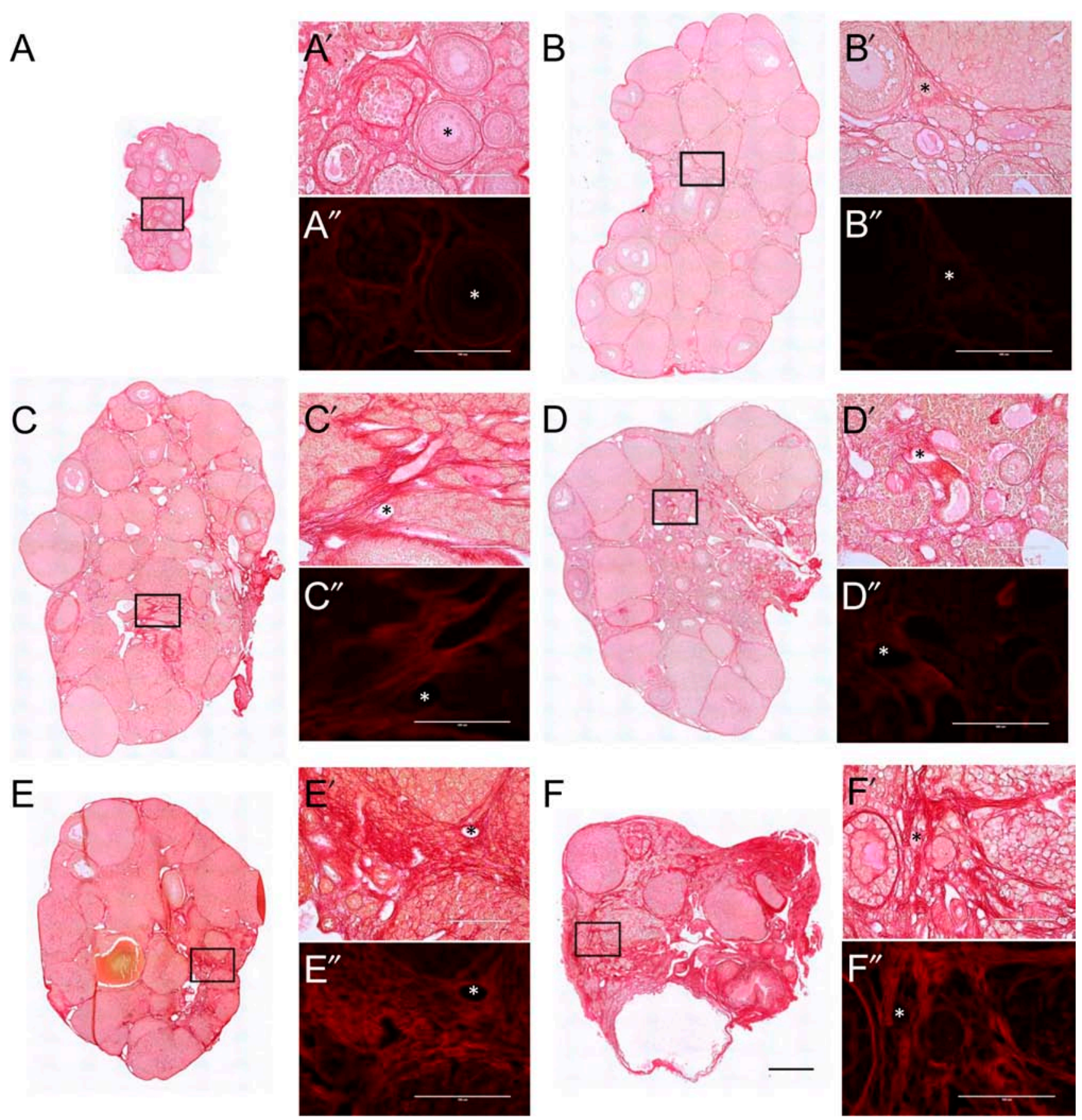

Figure 4 PSR staining expands throughout the ovarian stroma with advanced reproductive age. Scans of PSR-stained ovarian sections from (A) 6-week old, (B) 5-month old, (C) 7-month old, (D) 9-month old, (E) 18-month old and (F) 22-month old CD1 mice visualized by bright-field microscopy. The scale bar for images in $(A-F)$ is $0.4 \mathrm{~mm}$. $\left(A^{\prime}-F^{\prime}\right.$ and $\left.A^{\prime \prime}-F^{\prime \prime}\right)$ are higher magnification images from the specific regions of the ovarian stroma that are boxed in (A-F). Bright-field images are shown in $\left(A^{\prime}-F^{\prime}\right)$ and fluorescence images are shown in $\left(A^{\prime \prime}-F^{\prime \prime}\right)$. The fluorescence images were taken using a Texas Red LED Light cube with constant settings that were established for the 22-month old sample $\left(\mathrm{F}^{\prime \prime}\right)$. Therefore intensity differences across ages can be compared. Asterisks mark corresponding regions between bright-field and fluorescence images. Scale bars in $\left(\mathrm{A}^{\prime}-\mathrm{F}^{\prime}\right.$ and $\left.\mathrm{A}^{\prime \prime}-\mathrm{F}^{\prime \prime}\right)$ are $100 \mu \mathrm{m}$.

(Fig. 6E, F, G and H). Proline hydroxylation is a posttranslational modification found almost exclusively in connective tissue collagen, thereby hydroxyproline levels can be used as a surrogate measure of collagen metabolism and regulation (Reddy \& Enwemeka 1996,
McAnulty 2005). Proline hydroxylation allows the sharp twisting of the collagen helix and stabilizes the collagen structure. Notably, elevated hydroxyproline levels reflect higher collagen levels and are observed in fibrotic pathologies (McAnulty 2005). 

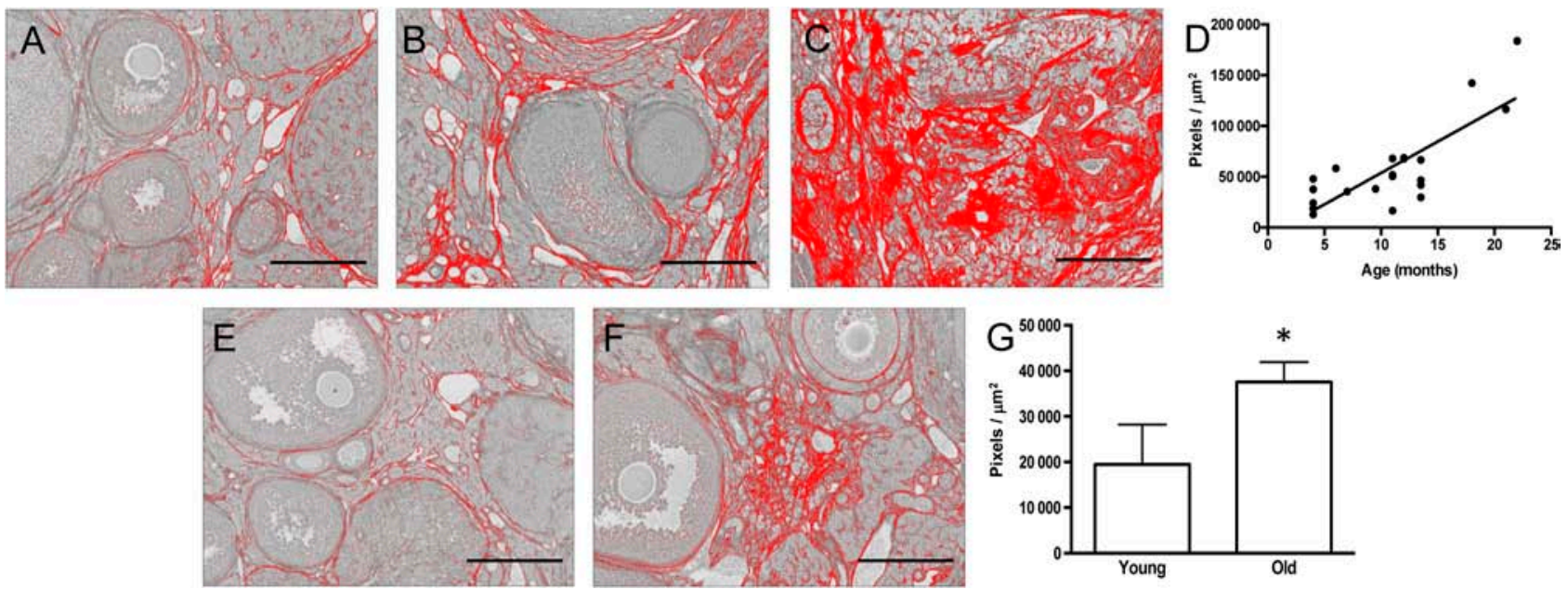

Figure 5 Fibrosis significantly increases in the ovarian stroma with advanced reproductive age in both CD1 and CB6F1 mice. Representative processed color threshold images of PSR-stained ovarian tissue sections used to quantify fibrosis from CD1 mice that were (A) 4 months old, (B) 13.5 months old and (C) 22 months old. (D) Graph showing the relationship between CD1 mouse age (months) and the average area of PSR-positive staining per ovarian section (pixels $/ \mu \mathrm{m}^{2}$ ). A significant linear relationship exists between these variables (Pearson's correlation, $P<0.0001$ and $R^{2}=0.6413$ ). Representative processed color threshold images of PSR-stained ovarian tissue sections used to quantify fibrosis from CB6F1 mice that were (E) 6-12 weeks old (young) and (F) 14-17 months old (old). (G) Graph comparing the average area of PSR-positive staining per ovarian section (pixels/ $\mu \mathrm{m}^{2}$ ) between reproductively young and old CB6F1 mice. The asterisk indicates a significant difference $(P=0.03)$. In all images, the red corresponds to PSR-positive staining, and scale bars are $200 \mu \mathrm{m}$.

We first validated the hydroxyproline assay using liver tissue from $\mathrm{CCl}_{4}$-treated and control mice, and as expected, we observed a higher collagen content in the $\mathrm{CCl}_{4}$-treated mice with known fibrosis (2-fold increase, data not shown). We then used this assay to extrapolate and compare the amount of collagen per $\mathrm{mg}$ of ovarian tissue from reproductively young and old mice. In each trial performed, we consistently observed a higher level of total collagen in ovaries from reproductively old mice compared to reproductively young mice, and this reproductive age-associated increase was significant in three of the four trials (Fig. 6E, F, G and H). When analyzed together, these trials represent an average 1.6-fold
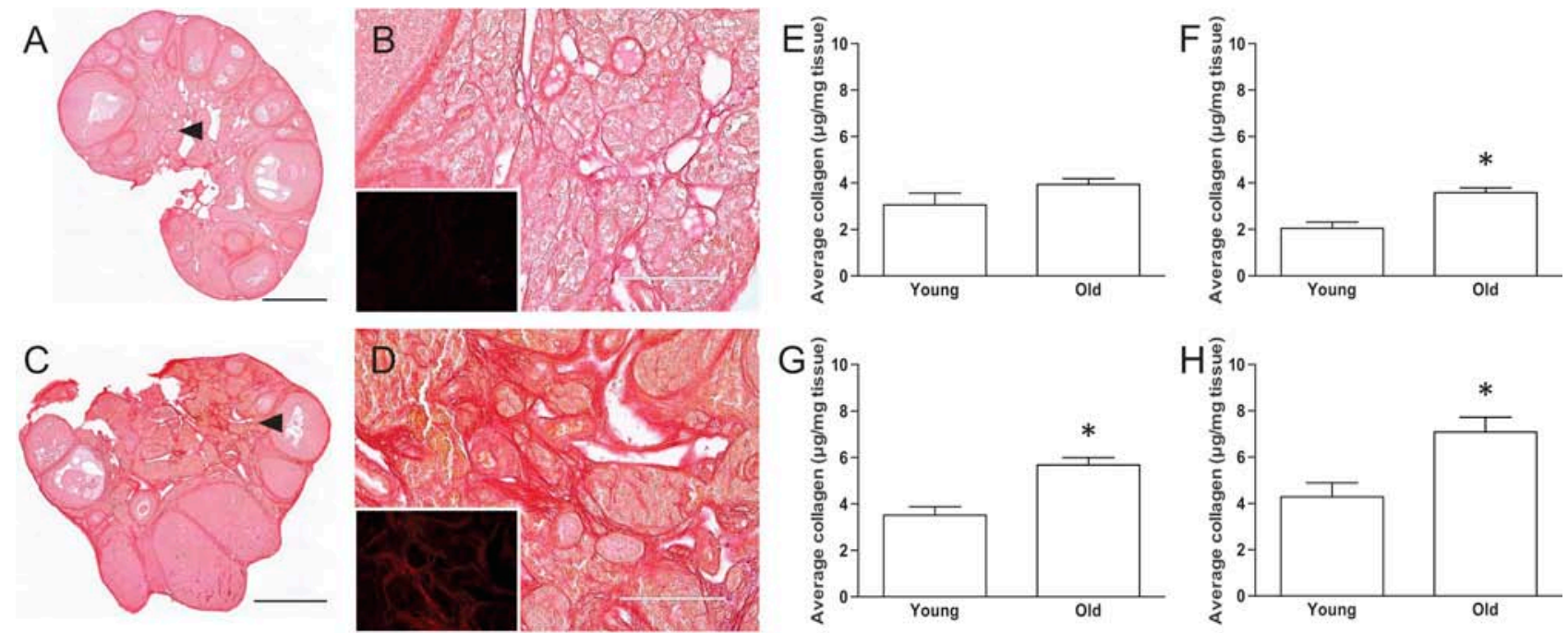

Figure 6 Reproductive age-associated increases in PSR staining correlate with increased ovarian hydroxyproline content. Scans of PSR-stained ovarian sections from (A and B) 6-12 weeks old (young) and (C and D) 14-17 months old (old) CB6F1 mice visualized by bright-field microscopy. Regions highlighted by the arrowheads in (A and C) are further magnified in (B and D). The insets in (B and D) show the corresponding fluorescence that is apparent when the PSR-stained tissue is viewed using epifluorescence with a Texas Red LED Light Cube. The fluorescence images were taken using constant settings that were established for the old ovary sample ( $D$, inset), and therefore, intensity differences between the age cohorts can be compared. Scale bars are $0.4 \mathrm{~mm}$ in (A and C) and $100 \mu \mathrm{m}$ in (B and D). (E, F, G and H) Extrapolation of collagen content $(\mu \mathrm{g} / \mathrm{mg}$ tissue) from hydroxyproline quantification in ovaries from reproductively young and old CB6F1 mice. Each graph represents data from a single trial. Asterisks mark significant differences (E: $P=0.255 ; \mathrm{F}: P=0.044 ; \mathrm{G}: P=0.004 ; \mathrm{H}: P=0.0186$ ). 
age-associated increase $(P=0.004)$. This increase in ovarian hydroxyproline content mirrors the results obtained with PSR staining and further confirms that the aging ovary includes a fibrotic microenvironment (Figs 4, 5 and 6).

\section{Ovarian fibrosis is associated with multinucleated macrophage giant cells}

Fibrosis is associated with inflammation, so we next investigated whether there were reproductive ageassociated changes in immune cells within the ovary (Libby 2007, Sziksz et al. 2015). Specifically we examined macrophages in ovarian sections from CB6F1 mice using an antibody against F4/80, a cell-surface membrane protein that is expressed at high levels in various mouse macrophages (Murray \& Wynn 2011). We found that there were similar numbers of F4/80positive cells in ovaries of reproductively young and old mice, comprising $23.9 \pm 1.7 \%$ and $25.4 \pm 0.83 \%$ of the total cell number, respectively (Fig. 7, inset, $P=0.45)$. This observation is consistent with the welldocumented role of macrophages in numerous ovarian processes including folliculogenesis, ovulation, and corpus luteum formation and regression (Wu et al. 2004). These macrophages had varying morphologies, including small spindle-shaped macrophages and
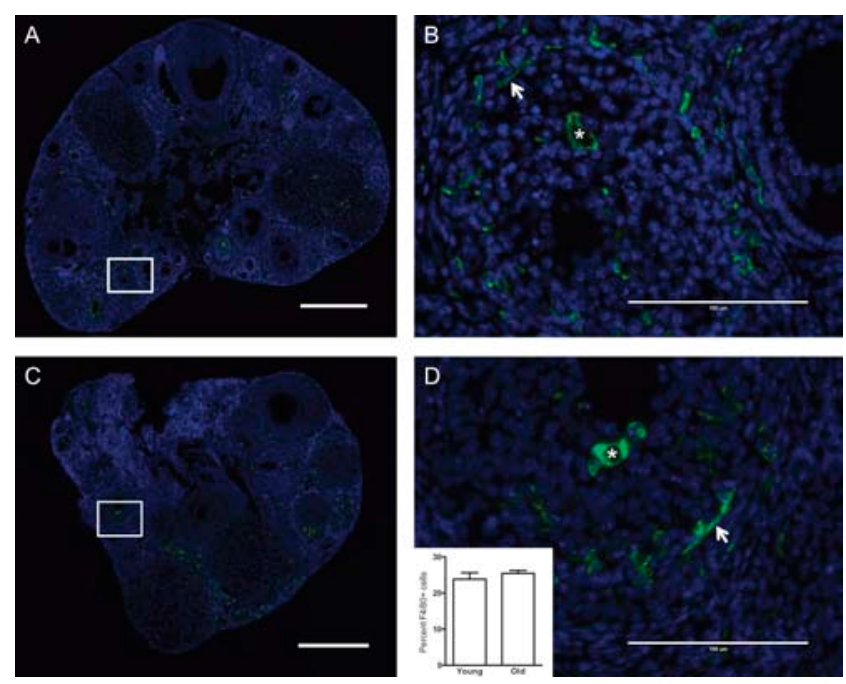

Figure 7 F4/80-positive macrophages are present in the ovarian stroma irrespective of reproductive age. Representative immunofluorescence images of ovarian sections from reproductively ( $\mathrm{A}$ and $\mathrm{B}$ ) young and ( $\mathrm{C}$ and $\mathrm{D})$ old CB6F1 mice stained with an F4/80-specific antibody. F4/80-positive cells are green and nuclei stained with DAPI are blue. Boxed regions in scans of entire ovarian sections (A and C) are further magnified in (B) and (D). Arrows highlight spindle-shaped macrophages, whereas asterisks highlight ovoid macrophages that have F4/80-positive staining around the entire cell surface. The inset in (D) shows the percentage of F4/80positive cells relative to total cell number in ovarian sections from reproductively young and old mice $(P=0.45)$. Scale bars are $0.4 \mathrm{~mm}$ in $(A$ and $C)$ and $100 \mu \mathrm{m}$ in (B and $D)$. ovoid macrophages, suggesting sub-populations with differing functions (Asano 2012) (Fig. 7B and D).

Although we did not observe any obvious ageassociated difference in the percentage of F4/80-positive cells in the ovarian stroma, we did document a unique cell population that was consistently observed in tissue from reproductively old CB6F1 mice relative to young mice (Fig. 8 and Table 1). Specifically, these cells stained a light brown with $\mathrm{H} \& \mathrm{E}$ and were enlarged, foamy and often multinucleated (Figs 8C, D, E, F and 9A, insets). By electron microscopy, these cells had vacuoles and inclusion bodies of various sizes indicative of phagocytosis of non-digestible material (Fig. 8E). Moreover, these cells exhibited high levels of autofluorescence relative to the surrounding tissue, which may be due to aggregated polymers resulting from oxidation of proteins and
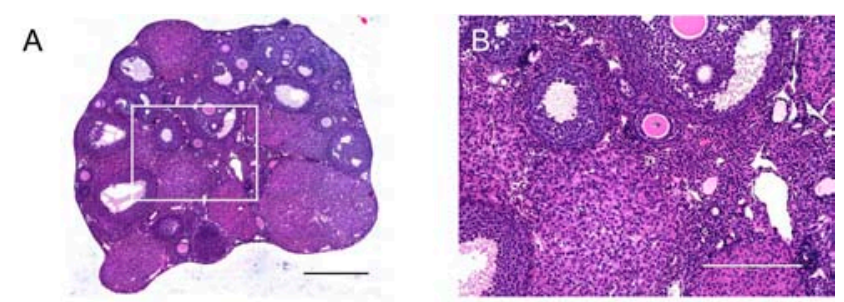

C
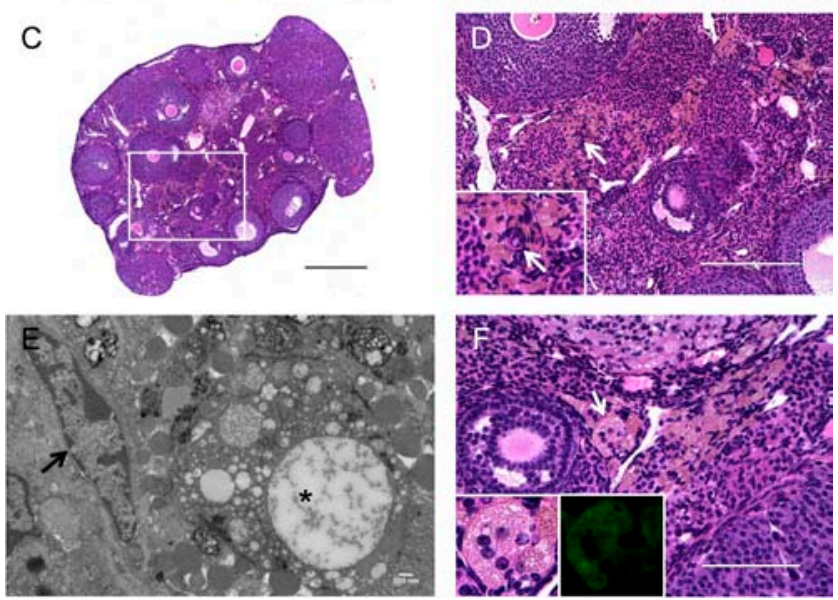

Figure 8 Multinucleated giant cells are present within the ovarian stroma of reproductively old mice. Hematoxylin and Eosin (H\&E) staining was performed on ovarian sections from (A and B) 6-12 weeks old (young) and (C, D and F) 14-17 months old (old) CB6F1 mice. (A) and $(\mathrm{C})$ are scans of the entire ovarian sections, and boxed regions are further magnified in (B) and (D) to highlight the ovarian stroma. In (C, $\mathrm{D}$ and $\mathrm{F}$ ), multinucleated giant cells appear foamy and stain brown by $\mathrm{H} \& \mathrm{E}$. The white arrows in (D) highlight a primordial follicle (inset) that localizes within this microenvironment containing multinucleated giant cells. (E) An electron micrograph of a thin ovarian tissue section from a reproductively old animal. The black arrow highlights a fibroblast with an elongated nucleus, and the asterisks highlight an enlarged giant cell with vacuoles and inclusion bodies. Another representative image of the ovarian stroma from reproductively old CB6F1 mice is shown in (F). The white arrow highlights a particular multinucleated giant cell in which 5 nuclei are clearly visible. This cell is magnified in the inset and its autofluorescent properties are shown (inset, right). Scale bars in ( $A$ and $C$ ) are $0.4 \mathrm{~mm}$, in (B and D) are $200 \mu \mathrm{m}, 500 \mathrm{~nm}$ in (E) and $100 \mu \mathrm{m}$ in (F). 
Table 1 Penetrance of macrophage giant cells in the ovarian stroma.

\begin{tabular}{lc}
\hline Age cohorts & $\begin{array}{c}\text { \# of mice with macrophage giant } \\
\text { cells/total }(\%)\end{array}$ \\
\hline CD1 mice & \\
$4-7$ months & $0 / 8(0)$ \\
$10-14$ months & $7 / 10(70)$ \\
$18-22$ months & $3 / 3(100)$ \\
CB6F1 mice & \\
$6-12$ weeks (young) & $0 / 10(0)$ \\
$14-17$ months (old) & $8 / 8(100)$ \\
\hline
\end{tabular}

lipids (Fig. 8F, inset) (Porta 2002). Cells with the same characteristics were also observed in the CD1 mouse strain and were associated with advanced reproductive age (Table 1 and data not shown). These cells were absent in mice $\leq 7$ months of age, present in $70 \%$ of mice between 10 and 14 months of age, and present in $100 \%$ of mice $\geq 18$ months of age (Table 1 ). Thus, these multinucleated giant cells correlate with increased age-associated fibrosis in the ovarian stroma.

The multinucleated giant cells were identified as macrophages because they stained positive for the F4/80 marker (Fig. 9B and D). The F4/80 staining at the cell periphery, however, was variable. In some enlarged macrophages that were still individualized, the staining was at the periphery (Fig. 9B, arrow), but in other cells within the cluster, F4/80 expression was reduced and absent from the cell surface (Fig. 9B and D). Due to their phagocytic function, multinucleated macrophage giant cells are enriched in polysaccharides that are distributed throughout the cytoplasm in small granules or in compact masses that stain positive with Periodic Acid Schiff (PAS) (Sobolev 1959). Using this histological stain, we demonstrated that the regions of the ovarian stroma that contain these multinucleated macrophage giant cells also stain intensely with PAS (Fig. 9B and D, insets). Taken together, these results suggest that macrophage fusion into multinucleated giant cells is a hallmark of the ovarian stroma during reproductive aging. Interestingly, these multinucleated giant cell macrophages were often observed in close proximity with cells that stained positive for $\alpha$-smooth muscle actin ( $\alpha$-SMA) (Fig. 9C), $\alpha$-SMA is a marker characteristic of fibroblast activation, which is associated with enhanced collagen secretion and, therefore, represents a critical transition in fibrogenesis (Hinz 2016). In lung and liver, profibrotic macrophages produce factors that regulate the proliferation, survival and activation of cells with fibrogenic potential (Murray \& Wynn 2011).

\section{Ovarian fibrosis is associated with an inflammatory microenvironment}

To verify whether reproductive age-related ovarian fibrosis is associated with increased inflammation, we interrogated the gene expression profiles of several genes involved in fibrosis and inflammation in whole ovaries isolated from reproductively young and old CB6F1 mice (Fig. 10). Specifically, we examined the expression of major pro-inflammatory cytokines that are also known to have pro-fibrotic functions, including $/ / 1 b, / / 6$ and Tnfa (Wynn \& Barron 2010, Wick et al. 2013, Sziksz et al. 2015) (Fig. 10A, B and C). These genes all showed higher levels of expression in ovaries from reproductively old mice relative to young mice, with $/ 11 b$ and Tnfa expression being significantly different between the age cohorts. In addition, we examined the expression pattern of genes involved in immune cell recruitment. $\mathrm{Ccl} 2$ encodes monocyte chemoattractant protein 1, which recruits monocytes to sites of inflammation where they ultimately differentiate into macrophages, and C Cl5 is involved in general leukocyte and fibroblast recruitment (Mantovani et al. 2004, Sahin \& Wasmuth 2013).
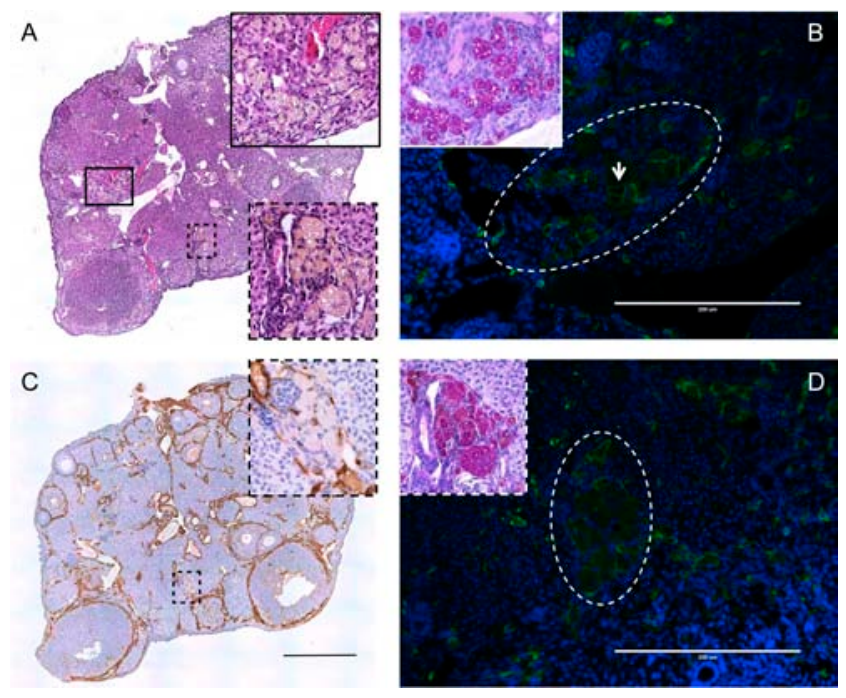

Figure 9 Multinucleated giant cells in the ovarian stroma are fused macrophages. (A) A representative hematoxylin and eosin (H\&E)stained ovarian section from a reproductively old CB6F1 mouse. The boxed regions (black solid and dashed), which highlight clusters of multinucleated giant cells, are further magnified in the insets. The black solid and dashed boxed regions are further examined in (B) and ( $C$ and $D)$, respectively. In ( $B$ and $D)$, ovarian sections were stained with an F4/80 antibody (green) and nuclei were visualized by DAPI (blue). The multinucleated giant cell clusters visible by H\&E in (A and C) are encircled by the white dashed lines in (B and D). The arrow in (B) highlights individual cells expressing F4/80 at the cell periphery in contrast to fused cells that lack complete borders (D). Insets in (B and D) show Periodic Acid Schiff (pink) staining within the clusters of multinucleated giant cells. Nuclei were visualized with hematoxylin (blue). In (C), an ovarian section was stained with an $\alpha$-Smooth Muscle Actin antibody (brown) and nuclei were visualized with hematoxylin (blue). The inset highlights the multinucleated giant cell cluster within the black dashed boxed region. Images in (A) and (C) are scans of the entire ovarian sections, whereas all other images highlight specific regions of interest in the ovarian stroma. All staining was done on sections that were between 5 and $30 \mu \mathrm{m}$ of each other to ensure that the same clusters of multinucleated giant cells were examined. The scales bars in (A and C) are $0.4 \mathrm{~mm}$ and in (B and D) are $200 \mu \mathrm{m}$. 

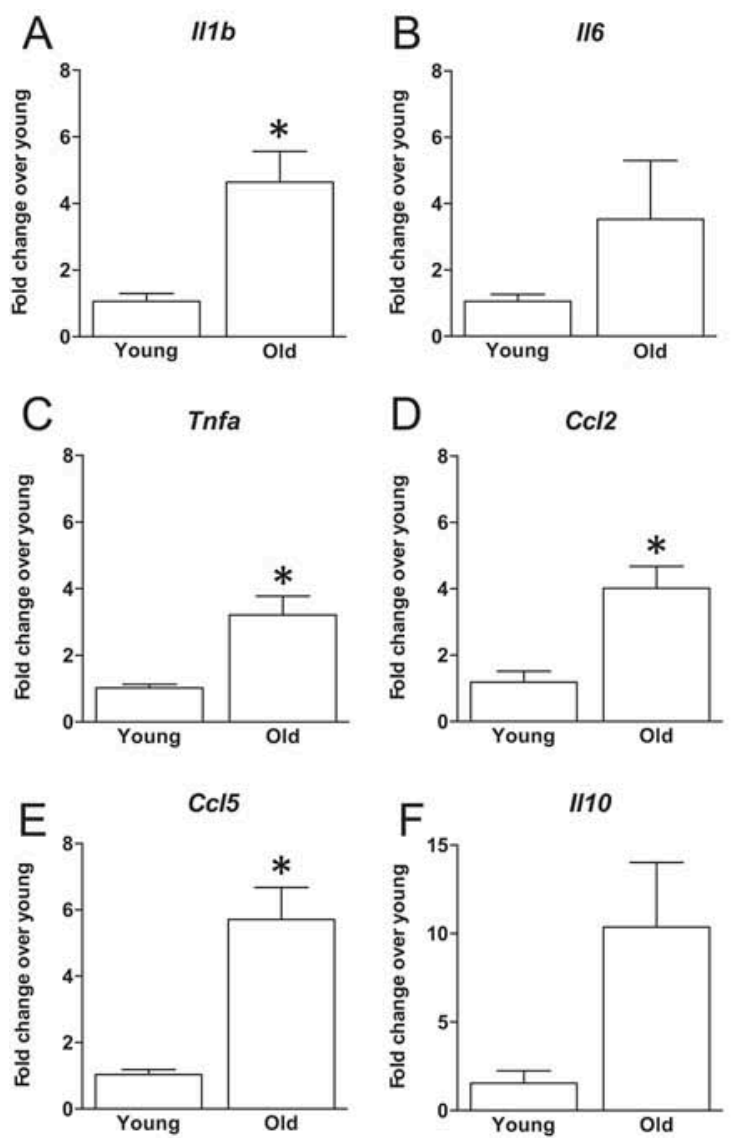

\begin{tabular}{|c|c|c|c|c|c|c|c|}
\hline POS & POS & NEG & NEG & GCSF & $\begin{array}{c}\text { GM- } \\
\text { CSF }\end{array}$ & IL-2 & IL-3 \\
\hline IL-4 & IL-5 & IL-6 & IL-9 & IL-10 & $\begin{array}{c}\text { IL-12 } \\
\text { p40/p70 }\end{array}$ & $\begin{array}{c}\text { IL-12 } \\
\text { p70 }\end{array}$ & IL-13 \\
\hline $\begin{array}{c}\text { IL- } \\
17 A\end{array}$ & $\begin{array}{c}\text { IFN- } \\
\text { gamma }\end{array}$ & $\begin{array}{c}\text { MCP1 } \\
\text { (CCL2) }\end{array}$ & MCP5 & $\begin{array}{c}\text { RANTES } \\
\text { (CCL5) }\end{array}$ & SCF & TNF RI & $\begin{array}{c}\text { TNF } \\
\text { alpha }\end{array}$ \\
\hline TPO & $\begin{array}{c}\text { VEGF- } \\
\text { A }\end{array}$ & BLANK & BLANK & BLANK & BLANK & BLANK & POS \\
\hline
\end{tabular}

$\mathrm{H}$
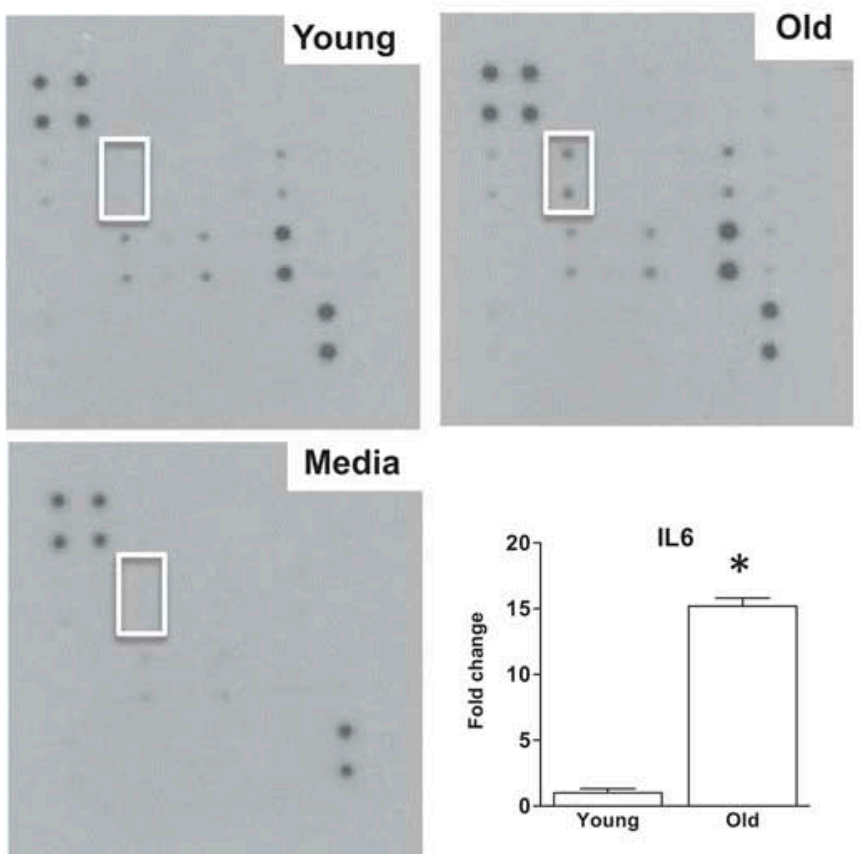

Figure 10 Reproductive aging is associated with an inflammatory ovarian microenvironment. Relative gene expression levels of (A) //1b, (B) //6, (C) CCl2, (D) Tnfa, (E) CCl5 and (F) //10 in ovaries from 6-12 weeks old (young) and 14-17 months old (old) CB6F1 mice. The data are shown as fold change expression in ovaries from reproductively old mice compared to reproductively young mice. Asterisks indicate significant differences (A: $P=0.002 \Delta \mathrm{Ct}, P=0.009$ fold change; $\mathrm{B}: P=0.07 \Delta \mathrm{Ct}, P=0.15$ fold change; $\mathrm{C}: P=0.02 \Delta \mathrm{Ct}, P=0.008$ fold change; $\mathrm{D}: P=0.002$ $\Delta \mathrm{Ct}, P=0.008$ fold change; E: $P=0.0002 \Delta \mathrm{Ct}, P=0.003$ fold change; $\mathrm{F}: P=0.02 \Delta \mathrm{Ct}, P=0.054$ fold change). (G) Schematic of the cytokines interrogated on the RayBio C-Series Mouse Cytokine Antibody Array C1. Antibodies in each column are printed in duplicate. $(\mathrm{H})$ Representative arrays probed with conditioned culture media from ovaries from reproductively young (top left) and old (top right) mice and control media only (bottom left). The white box highlights the spots on the arrays that correspond to IL-6. The relative expression of IL-6 in the conditioned culture media from ovaries isolated from reproductively young and old mice was quantified (bottom right), and the asterisks indicate a significant difference $(P=0.0024)$.

Both $\mathrm{CCl} 2$ and $\mathrm{Ccl} 5$ expression were significantly increased in ovaries from reproductively old mice relative to young counterparts (Fig. 10D and E). Finally, we examined the expression of $/ / 10$, which is typically considered an anti-inflammatory cytokine with anti-fibrotic functions (Sziksz et al. 2015) (Fig. 10F). Although $/ / 10$ expression was higher in ovaries from reproductively old mice compared to young mice, this difference was not significant. However, IL-10 inhibits TNF- $\alpha$ production, and thus, the observed increase in IL-10 may represent a countermeasure within the ovary to modulate fibrosis (Denys et al. 2002).

To further assess the inflammatory microenvironment of the aging ovary, we performed cytokine antibody arrays on conditioned culture media from ovaries isolated from reproductively young and old CB6F1 mice (Fig. 10G and H). We only cultured the ovaries for $2.5 \mathrm{~h}$ to avoid confounding results due to tissue ischemia and necrosis. Due to this short-term culture and the small amount of tissue, we only detected signals from five cytokines in the conditioned culture media, including IL-6, CCL2, CCL5, TNFR1 and IL12 p70 (Fig. 10H). Despite the limitations of this approach, however, we identified a prominent and significant 15 -fold increase in IL-6 secretion in ovaries from reproductively old mice compared to young mice (Fig. $10 \mathrm{H}$ ), which parallels the increase observed at the gene expression level (Fig. 10B). The cytokine expression and secretion data, along with the presence of multinucleated macrophage giant cells, are consistent with a highly inflammatory milieu within the aging ovary.

\section{Discussion}

In this study, we used PSR, a classic and highly specific histological dye for collagen I and III fibers, to 
evaluate fibrosis within ovarian tissue (Junqueira et al. 1979, Junqueira et al. 1978). Using various imaging approaches, we validated that PSR stains this ovarian ECM network a bright red and also exhibits strong red fluorescence and birefringence. Histopathologists commonly use PSR staining to evaluate degrees of fibrosis because this method is cost-effective, simple and consistent relative to other immunohistochemical, molecular and biochemical approaches to assess fibrosis (Coleman 2011). Using PSR staining, we determined that fibrosis within the ovarian stroma increased with advanced reproductive age in both CD1 and CB6F1 mouse strains, and this is consistent with the increased collagen levels that we quantified in ovaries from reproductively old animals using the biochemical hydroxyproline assay. In ovaries from the oldest animals ( $>20$ months of age), PSR staining was the most prominent, and in some cases, accounted for $>35 \%$ of the total ovarian area. This extensive fibrosis resembles what is observed in hepatocyte nodules in liver cirrhosis or in schistosome-induced granuloma formation (Bataller et al. 2011, Olveda et al. 2014, Mathurin \& Bataller 2015). Although ovarian fibrosis was most severe in the oldest animals, regions of fibrosis were evident within the tissue earlier. For example, we consistently documented fibrotic foci and multinucleated macrophage giant cells in the ovarian stroma in mice that were 14-17 months of age, which based on a linear extrapolation of age would roughly correspond to women who are approximately 38-45years of age. Importantly in both mice and humans, this period of the reproductive lifespan is already characterized by quantifiable changes in egg quality - namely a dramatic increase in the incidence of aneuploidy upwards of $60 \%$ (Jones \& Lane 2013). Thus, the changes in the ovarian microenvironment we observed are coincident with this known decrease in egg quality and may play a causative role.

Although it is evident that fibrosis increases in the ovarian stroma with advanced reproductive age, the mechanism by which this occurs is still unknown. The ovary is composed of several ECM matrices including the follicular basal lamina matrix, the cumulus-oocyte matrix and the stromal matrix (Irving-Rodgers et al. 2010). Through the processes of folliculogenesis and ovulation, the ovary undergoes constant cycles of connective tissue remodeling and wound healing, which require a complex interplay between matrix metalloproteinases (MMPs) and tissue inhibitors of metalloproteinases (TIMPs) (Curry \& Osteen 2001). The age-associated increase in fibrosis could be due to increased synthesis and deposition of collagen or other ECM components and/or altered post-translational modifications. Consistent with this possibility, we observed $\alpha$-SMA-positive cells in the ovarian stroma, which may correspond to a myofibroblast population that produces excess collagen (Hinz 2016). Alternatively, or in combination with increased ECM production, there may be an ageassociated change in the homeostasis of ECM through an imbalance of the activities of MMPs and TIMPs. Distinguishing between these various mechanisms is currently under investigation.

In addition to a prominent age-associated increase in fibrosis, we also documented increased expression of genes involved in immune cell recruitment that occurred concomitantly with the presence of a unique population of multinucleated macrophage giant cells in ovaries from reproductively old mice. Macrophages undergo fusion with other macrophages to form multinucleated giant cells and are a hallmark of chronic inflammation (Helming \& Gordon 2009, McNally \& Anderson 2011). Multinucleated giant cells are observed in cases of pathological infection, sarcoidosis, rheumatoid arthritis, certain neoplasias, and at sites of device or biomaterial implants (McNally \& Anderson 2011). Although the biological significance of macrophage fusion and multinucleation is not well understood, it has been proposed that this process enhances the phagocytic function of the cells through increased size, which allows extracellular degradation of large targets (Helming \& Gordon 2009). These multinucleated giant cells are reminiscent of osteoclasts, which have a significant capacity to degrade large areas of bone and other poorly degradable material (Helming \& Gordon 2009). Thus, this cell population in the aged ovary may be involved in resolving fibrotic regions of tissue. Interestingly, this population of cells in the ovary had variable F4/80 staining, with some cells exhibiting a peripheral pattern of expression while others showing decreased expression. The downregulation of F4/80 may reflect the activation status of this cohort of macrophages as has been observed in other examples of fibrotic progression and resolution (Ramachandran et al. 2012).

Macrophages are also major secretory cells that release cytokines, chemokines and growth factors that greatly influence proper follicle development (Tingen et al.2011). Our gene expression results demonstrate that there are increased levels of pro-inflammatory cytokines within the aging ovary. IL-6 secretion from the ovary was significantly increased with advanced reproductive age. This is of particular interest because serum IL- 6 levels increase in humans with age, and IL- 6 may mediate fibrosis by transcriptional activation of collagen or by stimulation of cytokines that upregulate collagen in an autocrine manner (Maggio et al. 2006, O'Reilly et al. 2013). Further defining the inflammatory milieu of the aging ovary and determining whether multinucleated macrophage giant cells are a cause or consequence of chronic inflammation are warranted and currently under investigation. Ultimately modulating pro-inflammatory pathways may be an important therapeutic avenue for prolonging reproductive lifespan because, for example, in a mouse knockout model of the pro-inflammatory 
cytokine IL- $1 \alpha$, the ovarian lifespan, pregnancy rate and litter size were all increased relative to age-matched controls (Uri-Belapolsky et al. 2014).

A similar population of enlarged macrophage cells was observed previously in the ovarian stroma of aged C57B/6J mice, thereby corroborating our findings in CB6F1 and CD1 mice (Asano 2012). These enlarged macrophages were also characterized by a frothy cytoplasm with polymorphic inclusion bodies and lysosomes, multiple nuclei, and intense autofluorescence (Asano 2012). It was also noted that there was a reproductive age-associated accumulation of non-heme iron in the ovarian stroma and that the subpopulation of enlarged macrophages had particularly high levels of non-heme ferric iron (NHF[III]), non-heme ferrous iron (NHF[II]) and oxidative stress (Asano 2012). This nonheme iron accumulation in the ovary with age may result from heme oxygenase-1-mediated heme degradation during follicular atresia and luteal regression (Asano 2012). Disruption of iron homeostasis in several organs, including the liver, heart and pancreas, leads to organ fibrosis (Ramm \& Ruddell 2010). Thus, it is tempting to speculate that iron accumulation in the aging ovary may be a mechanism driving fibrosis. In addition, whether these changes are regulated hormonally is also of great interest as aromatase-knockout mice have ovaries with significant follicle loss, a large influx of macrophages, and an increase in collagen deposition (Britt et al. 2000).

Taken together, we used a combination of imaging approaches to establish PSR staining as an important tool for studying fibrosis in the ovary and documented a prominent reproductive age-associated increase in fibrosis within the ovarian stroma that was coincident with a population of multinucleated macrophage giant cells and increased inflammation. Investigating how the extra-follicular ovarian microenvironment changes with age will not only improve our understanding of ovarian biology but also have broader implications for women's health. For example, ovarian fibrosis is a key feature of polycystic ovarian syndrome (PCOS), which is also accompanied by chronic low-grade inflammation (Bulut et al. 2015). Additionally, ovarian fibrosis is an unintended consequence of iatrogenic insults such as chemotherapy and radiation (Stroud et al. 2009). Quantifying ovarian fibrosis using methods, such as multi-modal magnetic resonance elastography, could therefore serve as a non-invasive metric of ovarian function (Wood et al. 2015). Furthermore, therapeutic strategies that target fibrotic remodeling may have a promising future in improving reproductive function in the context of age-related infertility, PCOS and fertility preservation.

\section{Supplementary data}

This is linked to the online version of the paper at http://dx.doi. org/10.1530/REP-16-0129.

\section{Declaration of interest}

The authors declare that there is no conflict of interest that could be perceived as prejudicing the impartiality of the research reported.

\section{Funding}

This work was supported by the Center for Reproductive Health After Disease (P50 HD076188 to F E D) the National Centers for Translational Research in Reproduction and Infertility (NCTRI), the Centers of Biomedical Research Excellence (P20 GM104936 to F E D), the National Institutes of Health, the National Center for Research Resources (P20 RR021940 to M T P), the National Institute of General Medical Sciences (P20 GM103549 to M T P), and the National Institute of Environmental Health Sciences 'Training Program in Environmental Toxicology' (T32 ES007079 to J M M). In addition, summer student research for this project was supported by the Kansas Institutional Development Award (IDeA) (P20 GM103418). The Electron Microscopy Research Laboratory and Anatomy/COBRE Confocal Imaging Facility at KUMC are supported in part by NIH COBRE 9P20GM104936. The JEOL JEM-1400 TEM used in the study was purchased with funds from S10RR027564. The Histology Core at KUMC is supported by NICHD P30 HD002528 (Kansas IDDRC).

\section{Acknowledgements}

We acknowledge John Kelsh for his initial observations, which inspired this study; Dr Aaron Varghese for contributing to literature searches and providing technical assistance; Dr Teresa K Woodruff for allowing us to use the LC-Polscope in her laboratory; and Dr Dale Abrahamson for useful discussions.

\section{References}

Alves A, Gritsch K, Sirieix C, Drevon-Gaillot E, Bayon Y, Clermont G, Boutrand JP \& Grosgogeat B 2015 Computerized histomorphometric study of the splenic collagen polymorphism: a control-tissue for polarization microscopy. Microscopy Research and Technique 78 900-907. (doi:10.1002/jemt.22553)

Asano Y 2012 Age-related accumulation of non-heme ferric and ferrous iron in mouse ovarian stroma visualized by sensitive non-heme iron histochemistry. Journal of Histochemistry and Cytochemistry $\mathbf{6 0}$ 229-242. (doi:10.1369/0022155411431734)

Bataller R, Rombouts K, Altamirano J \& Marra F 2011 Fibrosis in alcoholic and nonalcoholic steatohepatitis. Best Practice \& Research Clinical Gastroenterology 25 231-244. (doi:10.1016/j.bpg.2011.02.010)

Berkholtz CB, Lai BE, Woodruff TK \& Shea LD 2006 Distribution of extracellular matrix proteins type I collagen, type IV collagen, fibronectin, and laminin in mouse folliculogenesis. Histochemistry and Cell Biology 126 583-592. (doi:10.1007/s00418-006-0194-1)

Britt KL, Drummond AE, Cox VA, Dyson M, Wreford NG, Jones ME, Simpson ER \& Findlay JK 2000 An age-related ovarian phenotype in mice with targeted disruption of the Cyp 19 (aromatase) gene. Endocrinology 141 2614-2623. (doi:10.1210/endo.141.7.7578)

Broekmans FJ, Soules MR \& Fauser BC 2009 Ovarian aging: mechanisms and clinical consequences. Endocrine Reviews 30 465-493. (doi:10.1210/er.2009-0006)

Bulut G, Kurdoglu Z, Donmez YB, Kurdoglu M \& Erten R 2015 Effects of jnk inhibitor on inflammation and fibrosis in the ovary tissue of a rat 
model of polycystic ovary syndrome. International Journal of Clinical and Experimental Pathology 8 8774-8785.

Check JH, Jamison T, Check D, Choe JK, Brasile D \& Cohen R 2011 Live delivery and implantation rates of donor oocyte recipients in their late forties are similar to younger recipients. Journal of Reproductive Medicine 56 149-152.

Chiang T, Duncan FE, Schindler K, Schultz RM \& Lampson MA 2010 Evidence that weakened centromere cohesion is a leading cause of age-related aneuploidy in oocytes. Current Biology 20 1522-1528. (doi:10.1016/j.cub.2010.06.069)

Coleman R 2011 Picrosirius red staining revisited. Acta Histochemica 113 231-233. (doi:10.1016/j.acthis.2010.02.002)

Constandinou C, Henderson N \& Iredale JP 2005 Modeling liver fibrosis in rodents. Methods in Molecular Medicine 117 237-250. (doi:10.1385/ 1-59259-940-0:237)

Curry TE Jr \& Osteen KG 2001 Cyclic changes in the matrix metalloproteinase system in the ovary and uterus. Biology of Reproduction 64 1285-1296. (doi:10.1095/biolreprod64.5.1285)

Denic A, Glassock RJ \& Rule AD 2016 Structural and functional changes with the aging kidney. Advances in Chronic Kidney Disease 23 19-28. (doi:10.1053/j.ackd.2015.08.004)

Denys A, Udalova IA, Smith C, Williams LM, Ciesielski CJ, Campbell J, Andrews C, Kwaitkowski D \& Foxwell BM 2002 Evidence for a dual mechanism for IL-10 suppression of TNF-alpha production that does not involve inhibition of p38 mitogen-activated protein kinase or NF-kappa B in primary human macrophages. Journal of Immunology 168 4837-4845. (doi:10.4049/jimmunol.168.10.4837)

Dolber PC \& Spach MS 1993 Conventional and confocal fluorescence microscopy of collagen fibers in the heart. Journal of Histochemistry and Cytochemistry 41 465-469. (doi:10.1177/41.3.7679127)

Heffner LJ 2004 Advanced maternal age - how old is too old? New England Journal of Medicine 351 1927-1929. (doi:10.1056/NEJMp048087)

Helming L \& Gordon S 2009 Molecular mediators of macrophage fusion. Trends in Cell Biology 19 514-522. (doi:10.1016/j.tcb.2009.07.005)

Hinz B 2016 Myofibroblasts. Experimental Eye Research 142 56-70. (doi:10.1016/j.exer.2015.07.009)

Hirshfeld-Cytron JE, Duncan FE, Xu M, Jozefik JK, Shea LD \& Woodruff TK 2011 Animal age, weight and estrus cycle stage impact the quality of in vitro grown follicles. Human Reproduction 26 2473-2485. (doi:10.1093/humrep/der183)

Horn MA \& Trafford AW 2016 Aging and the cardiac collagen matrix: novel mediators of fibrotic remodelling. Journal of Molecular and Cellular Cardiology 93 175-185. (doi:10.1016/j.yjmcc.2015.11.005)

Hornick JE, Duncan FE, Sun M, Kawamura R, Marko JF \& Woodruff TK 2015 Age-associated alterations in the micromechanical properties of chromosomes in the mammalian egg. Journal of Assisted Reproduction and Genetics 32 765-769. (doi:10.1007/s10815-015-0453-y)

Irving-Rodgers HF, Hummitzsch K, Murdiyarso LS, Bonner WM, Sado Y, Ninomiya Y, Couchman JR, Sorokin LM \& Rodgers RJ 2010 Dynamics of extracellular matrix in ovarian follicles and corpora lutea of mice. Cell and Tissue Research 339 613-624. (doi:10.1007/s00441-009-0905-8)

Johnson JA, Tough S \& Society of Obstetricians and Gynaecologists of Canada 2012 Delayed child-bearing. Journal of Obstetrics and Gynaecology Canada 34 80-93. (doi:10.1016/S1701-2163(16)35138-6)

Jones KT \& Lane SI 2013 Molecular causes of aneuploidy in mammalian eggs. Development 140 3719-3730. (doi:10.1242/dev.090589)

Junqueira LC, Cossermelli W \& Brentani R 1978 Differential staining of collagens type I, II and III by Sirius Red and polarization microscopy. Archivum Histologicum Japonicum 41 267-274. (doi:10.1679/ aohc1950.41.267)

Junqueira LC, Bignolas G \& Brentani RR 1979 Picrosirius staining plus polarization microscopy, a specific method for collagen detection in tissue sections. Histochemical Journal 11 447-455. (doi:10.1007/ BF01002772)

Kapetanaki MG, Mora AL \& Rojas M 2013 Influence of age on wound healing and fibrosis. Journal of Pathology 229 310-322. (doi:10.1002/ path.4122)

Lattouf R, Younes R, Lutomski D, Naaman N, Godeau G, Senni K \& Changotade S 2014 Picrosirius red staining: a useful tool to appraise collagen networks in normal and pathological tissues. Journal of Histochemistry and Cytochemistry 62 751-758. (doi:10.1369/0022155414545787)
Libby P 2007 Inflammatory mechanisms: the molecular basis of inflammation and disease. Nutrition Reviews 65 S140-S146. (doi:10.1301/nr.2007.dec.S140-S146)

Maggio M, Guralnik JM, Longo DL \& Ferrucci L 2006 Interleukin-6 in aging and chronic disease: a magnificent pathway. Journals of Gerontology Series A: Biological Sciences and Medical Sciences 61 575-584. (doi:10.1093/gerona/61.6.575)

Mantovani A, Sica A, Sozzani S, Allavena P, Vecchi A \& Locati M 2004 The chemokine system in diverse forms of macrophage activation and polarization. Trends in Immunology 25 677-686. (doi:10.1016/ j.it.2004.09.015)

Mathurin P \& Bataller R 2015 Trends in the management and burden of alcoholic liver disease. Journal of Hepatology 62 S38-S46. (doi:10.1016/j.jhep.2015.03.006)

McAnulty RJ 2005 Methods for measuring hydroxyproline and estimating in vivo rates of collagen synthesis and degradation. Methods in Molecular Medicine 117 189-207.

McNally AK \& Anderson JM 2011 Macrophage fusion and multinucleated giant cells of inflammation. Advances in Experimental Medicine and Biology 713 97-111. (doi:10.1007/978-94-007-0763-4)

Mehta SB, Shribak M \& Oldenbourg R 2013 Polarized light imaging of birefringence and diattenuation at high resolution and high sensitivity. Journal of Optics 15 094007. (doi:10.1088/2040-8978/15/9/094007)

Merriman JA, Jennings PC, McLaughlin EA \& Jones KT 2012 Effect of aging on superovulation efficiency, aneuploidy rates, and sister chromatid cohesion in mice aged up to 15 months. Biology of Reproduction 8649. (doi:10.1095/biolreprod.111.095711)

Montes GS \& Junqueira LC 1991 The use of the Picrosirius-polarization method for the study of the biopathology of collagen. Memórias do Instituto Oswaldo Cruz 86 (Supplement 3) 1-11. (doi:10.1590/S007402761991000700002)

Murray PJ \& Wynn TA 2011 Protective and pathogenic functions of macrophage subsets. Nature Reviews Immunology 11 723-737. (doi:10.1038/nri3073)

Nagaoka SI, Hassold TJ \& Hunt PA 2012 Human aneuploidy: mechanisms and new insights into an age-old problem. Nature Reviews Genetics 13 493-504. (doi:10.1038/nrg3245)

Nielsen LF, Moe D, Kirkeby S \& Garbarsch C 1998 Sirius red and acid fuchsin staining mechanisms. Biotechnic \& Histochemistry 73 71-77. (doi:10.3109/10520299809140509)

O'Reilly S, Cant R, Ciechomska M \& van Laar JM 2013 Interleukin-6: a new therapeutic target in systemic sclerosis? Clinical \& Translational Immunology 2 e4. (doi:10.1038/cti.2013.2)

Olveda DU, Olveda RM, McManus DP, Cai P, Chau TN, Lam AK, Li Y, Harn DA, Vinluan ML \& Ross AG 2014 The chronic enteropathogenic disease schistosomiasis. International Journal of Infectious Diseases $\mathbf{2 8}$ 193-203. (doi:10.1016/j.ijid.2014.07.009)

Pascual M, Fernandez-Lizarbe S \& Guerri C 2011 Role of TLR4 in ethanol effects on innate and adaptive immune responses in peritoneal macrophages. Immunology and Cell Biology 89 716-727. (doi:10.1038/ icb.2010.163)

Porta EA 2002 Pigments in aging: an overview. Annals of the New York Academy of Sciences 959 57-65. (doi:10.1111/j.1749-6632.2002. tb02083.x)

Pritchard MT \& Nagy LE 2010 Hepatic fibrosis is enhanced and accompanied by robust oval cell activation after chronic carbon tetrachloride administration to Egr-1-deficient mice. American Journal of Pathology 176 2743-2752. (doi:10.2353/ajpath.2010.091186)

Pritchard MT, Cohen JI, Roychowdhury S, Pratt BT \& Nagy LE 2010 Early growth response-1 attenuates liver injury and promotes hepatoprotection after carbon tetrachloride exposure in mice. Journal of Hepatology 53 655-662. (doi:10.1016/j.jhep.2010.04.017)

Ramachandran P, Pellicoro A, Vernon MA, Boulter L, Aucott RL, Ali A, Hartland SN, Snowdon VK, Cappon A, Gordon-Walker TT et al. 2012 Differential Ly-6C expression identifies the recruited macrophage phenotype, which orchestrates the regression of murine liver fibrosis. PNAS 109 E3186-E3195. (doi:10.1073/ pnas.1119964109)

Ramm GA \& Ruddell RG 2010 Iron homeostasis, hepatocellular injury, and fibrogenesis in hemochromatosis: the role of inflammation in a noninflammatory liver disease. Seminars in Liver Disease 30 271-287. (doi:10.1055/s-0030-1255356) 
Reddy GK \& Enwemeka CS 1996 A simplified method for the analysis of hydroxyproline in biological tissues. Clinical Biochemistry 29 225-229. (doi:10.1016/0009-9120(96)00003-6)

Sahin H \& Wasmuth HE 2013 Chemokines in tissue fibrosis. Biochimica et Biophysica Acta 1832 1041-1048. (doi:10.1016/j. bbadis.2012.11.004)

Sangaralingham SJ, Wang BH, Huang L, Kumfu S, Ichiki T, Krum H \& Burnett JC Jr 2016 Cardiorenal fibrosis and dysfunction in aging: imbalance in mediators and regulators of collagen. Peptides $\mathbf{7 6}$ 108-114. (doi:10.1016/j.peptides.2016.01.004)

Schneider CA, Rasband WS \& Eliceiri KW 2012 NIH Image to Image): 25 years of image analysis. Nature Methods 9 671-675. (doi:10.1038/ nmeth.2089)

Sobolev SM 1959 [Result of histochemical studies on certain PAS-positive substances in macrophages]. Biulleten' eksperimental'no ́ biologii $i$ meditsiny 47 104-109.

Spandidos A, Wang X, Wang H, Dragnev S, Thurber T \& Seed B 2008 A comprehensive collection of experimentally validated primers for Polymerase Chain Reaction quantitation of murine transcript abundance. BMC Genomics 9 633. (doi:10.1186/1471-2164-9-633)

Spandidos A, Wang X, Wang H \& Seed B 2010 PrimerBank: a resource of human and mouse PCR primer pairs for gene expression detection and quantification. Nucleic Acids Research 38 D792-D799. (doi:10.1093/ nar/gkp1005)

Street JM, Souza AC, Alvarez-Prats A, Horino T, Hu X, Yuen PS \& Star RA 2014 Automated quantification of renal fibrosis with Sirius Red and polarization contrast microscopy. Physiological Reports 2 e12088. (doi:10.14814/phy2.12088)

Stroud JS, Mutch D, Rader J, Powell M, Thaker PH \& Grigsby PW 2009 Effects of cancer treatment on ovarian function. Fertility and Sterility $\mathbf{9 2}$ 417-427. (doi:10.1016/j.fertnstert.2008.07.1714)

Sziksz E, Pap D, Lippai R, Beres NJ, Fekete A, Szabo AJ \& Vannay A 2015 Fibrosis related inflammatory mediators: role of the IL-10 cytokine family. Mediators of Inflammation 2015 1-15. (doi:10.1155/2015/764641)

Tingen CM, Kiesewetter SE, Jozefik J, Thomas C, Tagler D, Shea L \& Woodruff TK 2011 A macrophage and theca cell-enriched stromal cell population influences growth and survival of immature murine follicles in vitro. Reproduction 141 809-820. (doi:10.1530/ REP-10-0483)

Treff NR, Krisher RL, Tao X, Garnsey H, Bohrer C, Silva E, Landis J, Taylor D, Scott RT, Woodruff TK et al. 2016 Next generation sequencingbased comprehensive chromosome screening in mouse polar bodies, oocytes, and embryos. Biology of Reproduction 94 76. (doi:10.1095/ biolreprod.115.135483)

Uri-Belapolsky S, Shaish A, Eliyahu E, Grossman H, Levi M, Chuderland D, Ninio-Many L, Hasky N, Shashar D, Almog T et al. 2014 Interleukin-1 deficiency prolongs ovarian lifespan in mice. PNAS 111 12492-12497. (doi:10.1073/pnas.1323955111)

Ushiki T 2002 Collagen fibers, reticular fibers and elastic fibers. A comprehensive understanding from a morphological viewpoint. Archives of Histology and Cytology 65 109-126. (doi:10.1679/ aohc.65.109)

Vogel B, Siebert H, Hofmann U \& Frantz S 2015 Determination of collagen content within picrosirius red stained paraffin-embedded tissue sections using fluorescence microscopy. MethodsX 2 124-134. (doi:10.1016/ j.mex.2015.02.007)

Wang X \& Seed B 2003 A PCR primer bank for quantitative gene expression analysis. Nucleic Acids Research 31 e154. (doi:10.1093/ nar/gng154)

Weber LW, Boll M \& Stampfl A 2003 Hepatotoxicity and mechanism of action of haloalkanes: carbon tetrachloride as a toxicological model. Critical Reviews in Toxicology 33 105-136. (doi:10.1080/ 713611034)

Whittaker P, Kloner RA, Boughner DR \& Pickering JG 1994 Quantitative assessment of myocardial collagen with picrosirius red staining and circularly polarized light. Basic Research in Cardiology 89 397-410. (doi:10.1007/BF00788278)

Wick G, Grundtman C, Mayerl C, Wimpissinger TF, Feichtinger J, Zelger B, Sgonc R \& Wolfram D 2013 The immunology of fibrosis. Annual Review of Immunology 31 107-135. (doi:10.1146/annurevimmunol-032712-095937)

Wood CD, Vijayvergia M, Miller FH, Carroll T, Fasanati C, Shea LD, Brinson LC \& Woodruff TK 2015 Multi-modal magnetic resonance elastography for noninvasive assessment of ovarian tissue rigidity in vivo. Acta Biomaterialia 13 295-300. (doi:10.1016/j.actbio. 2014.11.022)

Wu R, Van der Hoek KH, Ryan NK, Norman RJ \& Robker RL 2004 Macrophage contributions to ovarian function. Human Reproduction Update 10 119-133. (doi:10.1093/humupd/dmh011)

Wynn TA \& Barron L 2010 Macrophages: master regulators of inflammation and fibrosis. Seminars in Liver Disease 30 245-257. (doi:10.1055/s-0030-1255354)

Wynn TA \& Ramalingam TR 2012 Mechanisms of fibrosis: therapeutic translation for fibrotic disease. Nature Medicine 18 1028-1040. (doi:10.1038/nm.2807)

Received 10 March 2016

First decision 8 April 2016

Revised manuscript received 4 June 2016

Accepted 29 June 2016 